\title{
Exploiting Constructive Mutual Coupling in P2P MIMO by Analog-Digital Phase Alignment
}

\author{
Ang Li, Student Member, IEEE, and Christos Masouros, Senior Member, IEEE
}

\begin{abstract}
In this paper, we propose a joint analogdigital (A/D) beamforming scheme for the point-to-point multiple-input-multiple-output system, where we exploit mutual coupling by optimizing the load impedances of the transmit antennas. Contrary to the common conception that mutual coupling strictly harms the system performance, we show that mutual coupling can be beneficial by exploiting the concept of constructive interference. By changing the value of each load impedance for the antenna array based on convex optimization, the mutual coupling effect can be manipulated so that the resulting interference aligns constructively to the useful signal vector. We first prove that the full elimination of mutual coupling effect is not achievable solely by tuning the values of the antenna load impedances. We then introduce the proposed A/D scheme for both PSK and QAM modulations, where performance gains with respect to conventional techniques are obtained. The implementation of the proposed schemes is also discussed, where a lookup table can be built to efficiently apply the calculated load impedances. The numerical results show that the proposed schemes can achieve an improved performance compared to systems with fixed mutual coupling, especially when the antenna spacing is small.
\end{abstract}

Index Terms-MIMO, mutual coupling, constructive interference, optimization.

\section{INTRODUCTION}

$\mathbf{T}$ HE benefits of multiple-input-multiple-output (MIMO) systems have been widely acknowledged and extensively studied in recent years due to the performance gains over single-input-single-output (SISO) systems. One popular application of MIMO techniques is to employ spatial multiplexing to improve the system capacity by sending parallel data streams across multiple transmit antennas [1], [2]. At the receiver, these information streams can be separated by various signal processing techniques. Among receiver architectures, the maximum likelihood (ML) receiver can provide the optimal performance, but the computational complexity for ML is too high for its application in practice [3]. Therefore, linear receivers such as zero-forcing (ZF) and minimum meansquared-error (MMSE) that provide a sub-optimal performance

Manuscript received April 23, 2016; revised September 3, 2016 and November 16, 2016; accepted January 20, 2017. Date of publication January 24, 2017; date of current version March 8, 2017. This work was supported in part by the Royal Academy of Engineering, U.K., in part by the Engineering and Physical Sciences Research Council Project under Grant EP/M014150/1, and in part by the China Scholarship Council. The associate editor coordinating the review of this paper and approving it for publication was W. Gerstacker.

The authors are with the Department of Electronic and Electrical Engineering, University College London, London WC1E 7JE, U.K. (e-mail: ang.li.14@ucl.ac.uk; chris.masouros@ieee.org).

Color versions of one or more of the figures in this paper are available online at http://ieeexplore.ieee.org.

Digital Object Identifier 10.1109/TWC.2017.2657631 are proposed as alternative techniques [4], [5]. Compared to the ML receiver, linear receivers are more appealing for many applications due to the low cost of computational complexity. On the other hand, studies on precoding schemes have shown that interference can be exploited to benefit the system performance [6], [7], where the correlation rotation (also known as phase alignment) approach is proposed. By rotating the correlation of MIMO streams, the signals of interfering transmission are aligned to the signal of interest, which leads to a gain in the received SNR without investing additional power. Further studies on the constructive interference can be found in [8], [9] where it is exploited for transmit beamforming.

Many existing studies on receiver structures and preprocessing approaches of MIMO assume an uncorrelated Rayleigh flat fading channel, where there is no spatial correlation or mutual coupling effect between antenna elements. However, when the antenna spacing is small, these two effects cannot be neglected [10]-[12]. Therefore, investigations on the correlation and mutual coupling effect are of necessity. In [10], [11], the effect of spatial correlation and mutual coupling is studied when an increasing number of antennas is fitted in a fixed physical space. Experimental studies on the transmit correlation effect have been conducted in [12]-[14], and its impact on the system performance of MIMO has been investigated in [15]-[19]. Designs of the robust precoding schemes for the correlated channels are studied in [20]-[22]. As for the mutual coupling effect, in [23] the mutual coupling matrix is derived based on a $2 \times 2$ MIMO system and its effect on the MIMO capacity is also studied. Further studies on the mutual coupling can be found in [24]-[27], where the impact of mutual coupling effect in a Rician channel is investigated for the transmit beamforming in [24]. It is shown that for Rician channels, when the non-line-of-sight (NLOS) component dominates the correlated Rayleigh channel, the system capacity is low, while the system can achieve a higher capacity when the line-of-sight (LOS) component prevails. In [26], the effect of mutual coupling is studied, where it is shown that when the inter-spacing between antenna elements decreases, the mutual coupling effect becomes strong and has an inferior impact on the system performance. In [27], the mutual coupling is studied for MIMO systems at high SNR, where it is shown that in low correlation propagation environment, the mutual coupling effect will also degrade the system performance.

In order to compensate for the effect of the mutual coupling, a number of approaches have been proposed [28]-[30], most of which are based on the derivation of a compensation matrix. 
In [28], the mutual coupling compensation is realized by forming a compensation matrix, which is multiplied to the received signal vector, and experiments have validated the effectiveness of this scheme. In [29], the compensation matrix is derived based on the generalized scattering matrix of the antenna array and the spherical mode expansion. In [30], the mutual coupling compensation is studied for both transmitting and receiving antenna arrays, where the calculation of the compensation matrices is given. In [31]-[33], novel structures such as matching circuits are proposed to eliminate the mutual coupling effect. In [31], the mutual coupling effect at low terahertz $(\mathrm{THz})$ frequencies is studied, where a mantle cloaking method is applied for strip dipole antennas to reduce the mutual coupling effect. A novel structure is proposed in [32] to suppress the mutual coupling by adding a U-shaped microstrip, and its effectiveness has been validated. In [33], by adding parasitic elements to the antenna array, a reverse coupling effect is formulated and can alleviate the effect of mutual coupling. Other techniques that target at the mutual coupling compensation can be found in [34]-[36]. It should be noted that, however, most of the above existing techniques for mutual coupling compensation are not from a signal processing perspective.

Different from existing studies that aim at minimizing or compensating for the mutual coupling effect, in this paper we propose to exploit the mutual coupling effect to further improve the MIMO performance by exploiting the concept of constructive interference [6]. We focus on PSK modulated signals where this is done by aligning interference to the phase of the desired symbols, while the extension to QAM modulations is also investigated. We assume that each antenna element is equipped with a tunable load impedance (e.g. a varactor) such that the mutual coupling effect can be controlled by changing the values of each load impedance. While it will be shown that a full elimination of the mutual coupling effect solely by tuning the load values is not achievable, it is possible to exploit the mutual coupling to achieve phase alignment, where further performance gains can be obtained. We propose to employ the concept of constructive interference, where each transmit symbol after mutual coupling is enhanced and aligned to the phase of the desired transmit symbol. With the proposed approach, the mutual coupling effect can offer both amplitude scaling and angle rotation for each transmitted symbol, and as long as the received symbols after mutual coupling are located in the constructive region, a better detection performance can be achieved. We formulate the problem into a convex optimization problem, where the practical constraints on each load impedance is considered. The performance analysis of the proposed schemes is conducted in terms of the analytical probability of error. Furthermore, we also discuss the implementation of the proposed schemes in practice, where a lookup table can be built based on the transmit symbol vector such that the proposed schemes can be efficiently applied. We note that we focus on P2P links where typically small-scale MIMO systems are employed, such as in the cases of device-to-device (D2D) communications [37] and small cells [38] which have received increasing research attention recently and will play an important role in the future communication standards [39]. The proposed schemes are then best suited to these scenarios, where the size of the lookup table is acceptable, and the interference is present and can be exploited. Simulation results validate the performance gains by the proposed schemes, where for QPSK an SNR gain of $8 \mathrm{~dB}$ can be observed compared to MIMO systems with fixed mutual coupling, and even a $5 \mathrm{~dB}$ gain compared to the ideal MIMO systems without mutual coupling effect. Instead of compensating for the mutual coupling effect in existing literatures, the proposed schemes are shown to significantly improve the system performance.

For reasons of clarity, we summarize the contributions of this paper as:

1) We propose to exploit the mutual coupling effect for MIMO systems to further improve the system performance by means of analog processing with tunable antenna load impedances.

2) The problems are formulated by exploiting the concept of phase alignment and further transformed into optimization problems, where practical constraints are considered. Convex optimization can then be applied to solve the problems.

3) We characterize the fully-correlated channel model and mathematically analyze the system performance in terms of the analytical probability of error. We also discuss the practical implementation of the proposed schemes, where a lookup table can be built dependent on the transmit symbol vector.

The rest of this paper is organized as follows: Section II introduces the downlink point-to-point MIMO system and the channel model with spatial correlation and mutual coupling effect. Section III gives a detailed description of the proposed schemes that exploit the concept of constructive interference based on convex optimization. Section IV analyzes the system performance with the proposed schemes in terms of the probability of error. Section V discusses the practical implementation of the proposed schemes. Section VI presents numerical results to validate the superiority of the proposed techniques, and conclusions are given in Section VII.

Notations: $a$, a, and A denote scalar, vector and matrix, respectively. $\mathbb{E}\{\cdot\},(\cdot)^{T},(\cdot)^{H},(\cdot)^{-1},(\cdot)^{\dagger}$, and $\operatorname{tr}(\cdot)$ denote expectation, transpose, conjugate transpose, inverse, Moore-Penrose inverse and trace of a matrix respectively. $\|\cdot\|$ denotes the Frobenius norm, $\mathbf{I}$ is the identity matrix and $\mathbf{0}$ denotes a zero matrix or vector. $C^{n \times n}$ represents an $n \times n$ matrix in the complex set and $\mathbf{R}(k, u)$ denotes the element of the $k$ th-row and $u$ th-column in $\mathbf{R}$. diag $(\cdot)$ denotes the conversion into a diagonal matrix with the values on its main diagonal and vec $(\cdot)$ is the operation of transforming a diagonal matrix into a column vector. $\Re(\cdot)$ and $\Im(\cdot)$ denote the real part and imaginary part of a complex number, respectively.

\section{SySTEM MODEL}

In this section, the MIMO system is firstly introduced. Our focus is on compact antenna deployments at the transmitter side, which leads to both the mutual coupling and spatial correlation effect. Accordingly, we focus on the mutual coupling effect at the transmit antenna array, while the mutual coupling at the receiver side is considered fixed and therefore 


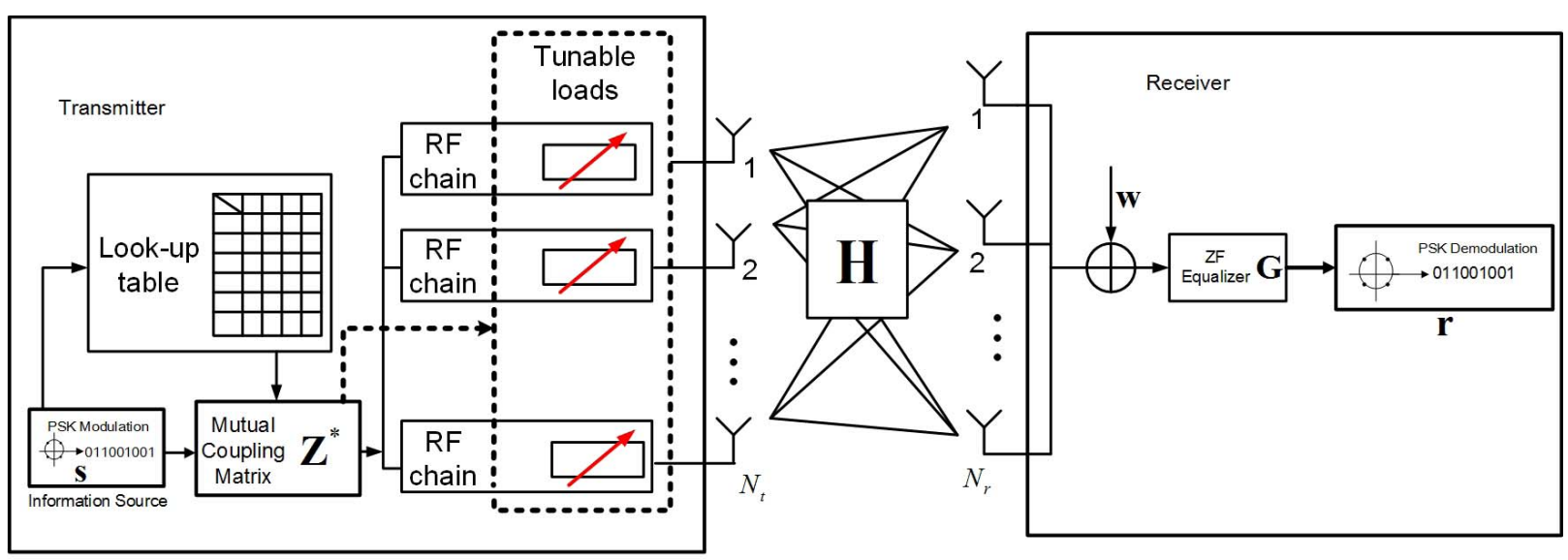

Fig. 1. Block diagram of the system model.

not included in the system model, which is in line with existing studies on mutual coupling [29], [31]-[34]. The spatial correlation effect is considered at both the transmit side and the receive side. In the following, we present the channel model with spatial correlation and mutual coupling.

\section{A. System Model}

We consider the model of a time-discrete point-topoint (P2P) MIMO channel with $N_{t}$ antennas at the transmitter and $N_{r}$ antennas at the receiver, as shown in Fig. 1, where $N_{r} \geq N_{t}$. There is a total number of $N_{t}$ streams, and the transmitted signal vector is formed from the constellation points of a PSK modulation, which is denoted as $\mathbf{s} \in C^{N_{t} \times 1}$. Each data symbol satisfies $\left\|s_{i}\right\|^{2}=1$, where a fixed power per symbol is maintained. The extension to QAM modulations will be discussed in Section III. With mutual coupling effect considered at the transmitter, the effective channel is composed by a mutual coupling matrix $\mathbf{Z} \in C^{N_{t} \times N_{t}}$ followed by the propagation channel $\mathbf{H} \in C^{N_{r} \times N_{t}}$. The received signal vector can then be obtained as

$$
\mathbf{y}=\mathbf{H Z} \mathbf{s}+\mathbf{w}
$$

where $\mathbf{w}$ is the additive Gaussian noise vector with $\mathbf{w} \sim C N\left(\mathbf{0}, \sigma^{2} \cdot \mathbf{I}\right)$. We assume full channel state information at the receiver side. While we do not consider the mutual coupling at the receiver side, we note that the proposed schemes can still be applied to the cases where there exist mutual coupling at the receiver by considering the effective channel $\hat{\mathbf{H}}=\mathbf{Z}_{r} \mathbf{H}$, where we denote $\mathbf{Z}_{r}$ as the mutual coupling matrix at the receiver side. At the receiver, the signal vector is linearly filtered by the equalizer $\mathbf{G} \in C^{N_{t} \times N_{r}}$, and the estimation for the intended data vector is then obtained as

$$
\mathbf{r}=\mathbf{G y}=\mathbf{G H Z s}+\mathbf{G w}
$$

For simplicity we assume a ZF equalizer given by

$$
\mathbf{G}=\left(\mathbf{H}^{H} \mathbf{H}\right)^{-1} \mathbf{H}^{H},
$$

in which case the signal at the output of the equalizer is given as

$$
\mathbf{r}=\mathbf{Z s}+\hat{\mathbf{w}}
$$

where $\hat{\mathbf{w}}=\mathbf{G w}$ is the noise vector after equalization. We note that the approaches detailed below can be straightforwardly extended to other equalizers by considering the resulting effective channel after equalization.

\section{B. Spatially Correlated Channel Model}

When the antenna spacing is small, the spatial correlation effect between antenna elements arise and should not be neglected in the channel model. As a P2P MIMO model is considered in this paper, therefore a fully-correlated NLOS Rayleigh flat fading channel is applied, where the spatial correlation is considered at both the transmitter and receiver. Following [40]-[42], we model the channel as

$$
\mathbf{H}=\mathbf{A}_{r} \mathbf{H}_{\alpha} \mathbf{A}_{t}^{H} \text {. }
$$

In (5), $\mathbf{H}_{\alpha}$ is a diagonal matrix with complex Gaussian distributed elements and is given by

$$
\mathbf{H}_{\alpha}=\frac{1}{\sqrt{M}} \cdot \operatorname{diag}\left(\alpha_{1}, \alpha_{2}, \ldots, \alpha_{M}\right),
$$

where $M$ is the number of random and independent rays, also known as the number of scatters in the wireless environment. $\alpha_{m}, m \in\{1,2, \ldots, M\}$ is the complex Rayleigh channel coefficient and each $\alpha_{m} \sim C N(0,1) . \mathbf{A}_{r} \in C^{N_{r} \times M}$ and $\mathbf{A}_{t} \in C^{N_{t} \times M}$ denote the receiver-side and transmitter-side steering matrices that contain $M$ steering vectors of the antenna array. For uniform linear arrays (ULAs), as assumed in this paper, $\mathbf{A}_{r}$ and $\mathbf{A}_{t}$ can be expressed as

$$
\begin{aligned}
\mathbf{A}_{r} & =\left[\mathbf{a}_{r}\left(\phi_{1}\right), \mathbf{a}_{r}\left(\phi_{2}\right), \ldots, \mathbf{a}_{r}\left(\phi_{M}\right)\right], \\
\mathbf{A}_{t} & =\left[\mathbf{a}_{t}\left(\varphi_{1}\right), \mathbf{a}_{t}\left(\varphi_{2}\right), \ldots, \mathbf{a}_{t}\left(\varphi_{M}\right)\right],
\end{aligned}
$$

where $\mathbf{a}_{r}\left(\phi_{m}\right) \in \mathcal{C}^{N_{r} \times 1}$ and $\mathbf{a}_{t}\left(\varphi_{m}\right) \in C^{N_{t} \times 1}$ of ULAs can be expressed as

$$
\begin{aligned}
& \mathbf{a}_{r}\left(\phi_{m}\right)=\left[1, e^{j 2 \pi d_{r} \sin \phi_{m}}, \ldots, e^{j 2 \pi\left(N_{r}-1\right) d_{r} \sin \phi_{m}}\right]^{T}, \\
& \mathbf{a}_{t}\left(\varphi_{m}\right)=\left[1, e^{j 2 \pi d_{t} \sin \varphi_{m}}, \ldots, e^{j 2 \pi\left(N_{t}-1\right) d_{t} \sin \varphi_{m}}\right]^{T} .
\end{aligned}
$$


In (8), $\phi_{m}$ and $\varphi_{m}, m \in\{1,2, \ldots, M\}$ denote the angles of arrival (AoAs) and angles of departure (AoDs) with the assumption that both $\phi_{m}$ and $\varphi_{m}$ follow a uniform distribution in $[-\pi, \pi] . d_{r}$ and $d_{t}$ are the equidistant antenna spacing normalized by the carrier wavelength for the receive and transmit antenna array, respectively.

\section{Modeling of the Mutual Coupling Effect}

Based on [11], [23], the mutual coupling matrix with tunable load impedances can be derived and defined as

$$
\mathbf{Z}=\left[z_{A} \cdot \mathbf{I}+\operatorname{diag}\left(\mathbf{z}_{L}\right)\right]\left[\boldsymbol{\Gamma}+\operatorname{diag}\left(\mathbf{z}_{L}\right)\right]^{-1},
$$

where $z_{A}$ denotes the antenna impedance that is considered as constant in this paper and $\mathbf{z}_{L}=\left[z_{L_{1}}, z_{L_{2}}, \ldots, z_{L_{N_{t}}}\right]^{T}$ is the load impedance vector to be optimized. $\Gamma$ is the mutual impedance matrix that can be expressed as

$$
\boldsymbol{\Gamma}=\left[\begin{array}{ccccc}
z_{A} & z_{m_{1}} & z_{m_{2}} & \cdots & z_{m_{N_{t}-1}} \\
z_{m_{1}} & z_{A} & z_{m_{1}} & \ddots & \vdots \\
z_{m_{2}} & z_{m_{1}} & \ddots & \ddots & z_{m_{2}} \\
\vdots & \ddots & \ddots & \ddots & z_{m_{1}} \\
z_{m_{N_{t}-1}} & \cdots & z_{m_{2}} & z_{m_{1}} & z_{A}
\end{array}\right] .
$$

In (10), $z_{m_{k}}$ denotes the mutual impedance of two antenna elements with the distance of $k \cdot d_{t}$ and $k \in\left\{1,2, \ldots, N_{t}-1\right\}$. The value of $z_{A}$ and $z_{m_{k}}$ can be obtained by the induced electromagnetic-field (EMF) method based on the transmit antenna spacing $d_{t}$ of the transmit antenna array (refer to Appendix).

The proposed techniques are then based on exploiting the mutual coupling between the transmit antennas by optimizing the values of each load impedance $z_{L_{i}}$.

\section{Proposed Schemes based on Constructive INTERFERENCE EXPLOITATION}

In this section, we firstly prove that the full elimination of mutual coupling effect is not achievable by solely tuning the load impedances, followed by the proposed schemes that exploit the mutual coupling to achieve phase alignment based on convex optimization. Before introducing the proposed schemes, for notational simplicity we first rewrite (9) as

$$
\mathbf{Z}=\mathbf{Z}_{d}\left(\mathbf{Z}_{d}+\tilde{\Gamma}\right)^{-1}
$$

where $\mathbf{Z}_{d}$ is the diagonal matrix and can be expressed

$$
\mathbf{Z}_{d}=\operatorname{diag}\left(z_{A}+z_{L_{1}}, z_{A}+z_{L_{2}}, \ldots, z_{A}+z_{L_{N_{t}}}\right)
$$

and $\tilde{\boldsymbol{\Gamma}}$ is obtained from $\boldsymbol{\Gamma}$ with all the diagonal elements being zero, given by

$$
\tilde{\boldsymbol{\Gamma}}=\left[\begin{array}{ccccc}
0 & z_{m_{1}} & z_{m_{2}} & \cdots & z_{m_{N_{t}-1}} \\
z_{m_{1}} & 0 & z_{m_{1}} & \ddots & \vdots \\
z_{m_{2}} & z_{m_{1}} & \ddots & \ddots & z_{m_{2}} \\
\vdots & \ddots & \ddots & \ddots & z_{m_{1}} \\
z_{m_{N_{t}-1}} & \ldots & z_{m_{2}} & z_{m_{1}} & 0
\end{array}\right] .
$$

Before being transmitted through the wireless channel, the signal vector is affected by the effect of mutual coupling, which is represented by the multiplication of the mutual coupling matrix. Therefore, we can regard the mutual coupling matrix as a linear scaling matrix that manipulates and scales the transmit signal vector, and the equivalent signal vector to be transmitted becomes

$$
\tilde{\mathbf{s}}=\mathbf{Z s}=\mathbf{Z}_{d}\left(\mathbf{Z}_{d}+\tilde{\boldsymbol{\Gamma}}\right)^{-1} \mathbf{s} .
$$

Based on the knowledge of linear algebra theory, the linear scaling of a vector can be equivalently represented by the vector multiplied by a diagonal matrix, and therefore $\tilde{\mathbf{s}}$ can also be expressed as

$$
\tilde{\mathbf{s}}=\operatorname{diag}\left(\lambda_{1}, \lambda_{2}, \ldots, \lambda_{N_{t}}\right) \mathbf{s},
$$

where $\lambda_{i}$ is the scaling factor for each transmit symbol $s_{i}$ and the property of $\lambda_{i}$ is dependent on the different schemes to be introduced in the following. It should be noted that the mathematical representation of (15) does not necessarily mean that there is no interference between transmit symbols, because each $\lambda_{i}$ can be a complex number. For different schemes, there are different constraints on the phase of each $\lambda_{i}$, which will be introduced in the following. Then, based on (14) and (15), we can obtain

$$
\mathbf{Z}_{d}\left(\mathbf{Z}_{d}+\tilde{\mathbf{\Gamma}}\right)^{-1} \mathbf{s}=\operatorname{diag}\left(\lambda_{1}, \lambda_{2}, \ldots, \lambda_{N_{t}}\right) \mathbf{s} .
$$

With

$$
\boldsymbol{\Lambda}=\left[\lambda_{1}, \lambda_{2}, \ldots, \lambda_{N_{t}}\right]^{T}
$$

and further transformation, (16) can be rewritten as

$$
\begin{aligned}
\mathbf{s} & =\left(\mathbf{Z}_{d}+\tilde{\mathbf{\Gamma}}\right) \mathbf{Z}_{d}^{-1} \operatorname{diag}(\boldsymbol{\Lambda}) \mathbf{s} \\
& \Rightarrow[\operatorname{diag}(\mathbf{\Lambda})-\mathbf{I}] \mathbf{s}+\tilde{\mathbf{\Gamma}} \mathbf{Z}_{d}^{-1} \operatorname{diag}(\mathbf{\Lambda}) \mathbf{s}=\mathbf{0},
\end{aligned}
$$

which reveals the relationship between each scaling factor $\lambda_{i}$ and the load impedance matrix $\mathbf{Z}_{d}$. Accordingly, two different design approaches for $\mathbf{Z}_{d}$ are explored and compared in the following.

\section{A. Full Elimination of the Mutual Coupling Effect}

We firstly consider the signal processing technique that fully eliminates the mutual coupling effect such that $\tilde{\mathbf{s}}=\mathbf{s}$. According to (15), this is equivalent to

$$
\lambda_{i}=1, \quad \forall i \in\left\{1,2, \ldots, N_{t}\right\} .
$$

By substituting (19) into (18), we can obtain the following

$$
\tilde{\mathbf{\Gamma}} \mathbf{Z}_{d}^{-1} \mathbf{s}=\mathbf{0},
$$

which means that for a particular transmit symbol vector $\mathbf{s}$, if we can adjust each value of the load impedance $z_{L_{i}}$ to satisfy (20), the effect of mutual coupling effect can be fully eliminated. Noting that $\mathbf{Z}_{d}$ is a diagonal matrix and with some transformation, (20) can be further transformed into

$$
\tilde{\boldsymbol{\Gamma}} \operatorname{diag}(\mathbf{s}) \operatorname{vec}\left(\mathbf{Z}_{d}^{-1}\right)=\mathbf{0},
$$

which leads to the following proposition. 
Proposition: It is not possible to fully eliminate the mutual coupling effect by changing the values of each load impedance $z_{L_{i}}$.

Proof: (21) can be regarded as a feasibility problem where we need to obtain the value of each load impedance to satisfy this equation for a pre-determined transmit symbol vector $\mathbf{s}$, which is equivalent to finding non-zero solutions for a linear system $\mathbf{A x}=0$. For this problem, $\mathbf{x}=\operatorname{vec}\left(\mathbf{Z}_{d}^{-1}\right)$ is a column vector and $\mathbf{A}=\tilde{\boldsymbol{\Gamma}}$ diag $(\mathbf{s})$. Based on linear algebra theory, the condition for such a linear system to have a non-zero solution is $\operatorname{det}(\mathbf{A})=0$, which means

$$
\operatorname{det}[\tilde{\boldsymbol{\Gamma}} \operatorname{diag}(\mathbf{s})]=0 .
$$

(22) can then be further transformed into

$$
\operatorname{det}(\tilde{\boldsymbol{\Gamma}})=0 \quad \text { or } \operatorname{det}[\operatorname{diag}(\mathbf{s})]=0 .
$$

Since diag (s) is a diagonal matrix and the determinant of $\tilde{\boldsymbol{\Gamma}}$ in (13) is also not zero, it is not possible for (21) to have non-zero solutions.

Remark: Based on the proposition, (21) can only have zero solutions. Then, with the definition of $\mathbf{Z}_{d}$ in (12), this means that the value of $z_{L_{i}}$ will be extremely large in practice such that $\mathbf{Z}_{d}^{-1}$ can approach $\mathbf{0}$. In this case, $z_{A}$ and $z_{m_{k}}$ will be very small compared to $z_{L_{i}}$ and therefore can be negligible. Then, (9) will be transformed into

$$
\begin{aligned}
\mathbf{Z} & =\left[z_{A} \cdot \mathbf{I}+\operatorname{diag}\left(\mathbf{z}_{L}\right)\right]\left[\boldsymbol{\Gamma}+\operatorname{diag}\left(\mathbf{z}_{L}\right)\right]^{-1} \\
& \approx \operatorname{diag}\left(\mathbf{z}_{L}\right)\left[\operatorname{diag}\left(\mathbf{z}_{L}\right)\right]^{-1}=\mathbf{I} .
\end{aligned}
$$

However, this case is not feasible in practice, as an extremely large value of the load impedance will result in an extremely small current in the transmitting antenna, which then leads to a very small power transfer efficiency [43], [44].

\section{B. Constructive Interference and Constructive Region}

While the full elimination of mutual coupling is not achievable, it is possible to exploit the concept of constructive interference to further improve the system performance. Constructive interference is defined as the interference that pushes the received symbols away from the decision thresholds of the modulation constellation [6], [7]. The exploitation of constructive interference to benefit the system performance is firstly introduced in [6], where the angles of the interfering signals are controlled and rotated such that they are strictly aligned to the angles of the transmit symbols of interest to achieve constructive interference. Furthermore, the concept of constructive region is introduced in [8], [9], where it is shown that the interfering signals may not be necessarily strictly aligned to the symbols of interest to obtain performance benefits, and that as long as the resulting interfered transmit symbols are located in the constructive region, the distance to the detection threshold is increased for the received symbols and therefore there will be a performance gain. To illustrate this intuitively, in Fig. 2 the constructive interference region [8], [9] is depicted, denoted by the green area in the constellation, where QPSK is employed as the example to demonstrate these two effects. For simplicity, here we focus

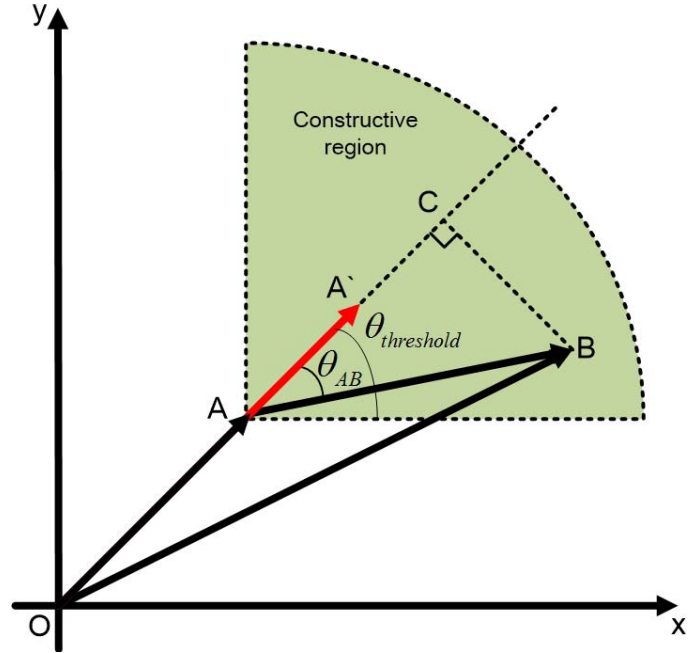

Fig. 2. Constructive Interference and Constructive Region.

on one quarter of the QPSK constellation corresponding to the $(1+j)$ constellation point, where the axes shown are the detection thresholds for the constellation points. While we mostly focus on QPSK in this paper, the extensions to other PSK modulations are also applicable [6], [9], and for QAM modulations the constructive interference can be exploited for the outer constellation points [45]-[48]. In the following, the proposed schemes to achieve phase alignment for PSK and QAM modulations are introduced, respectively.

\section{Exploiting Mutual Coupling for PSK Modulations}

We study the case where the interfered transmit symbols are located in the constructive region, and we firstly focus on PSK modulations. Then in Fig. 2, $\overrightarrow{O A}$ is the original information symbol, and here we assume that $\overrightarrow{A B}$ is the interfering signal for the transmit symbol of interest. With this interfering signal, the resulting received symbol will be $\overrightarrow{O B}$. As can be observed from Fig. 2, as long as the node B is located in the constructive region, the Euclidean distance of the received symbol $\overrightarrow{O B}$ to the detection threshold is increased with respect to the nominal constellation point $\overrightarrow{O A}$, and an improved performance can be achieved. Furthermore, the exploitation of constructive region relaxes the strict angle requirement for manipulating the interfering signals, and more importantly relaxes the value range of each load impedance for the proposed techniques to be introduced. Accordingly, different from the case in Section III-A where the full elimination is not possible, the exploitation of mutual coupling effect for constructive interference can be achieved. We refer the reader to [9], [45], [49], [50] for further details on the constructive region. Based on the above description and Fig. 2, we denote $\overrightarrow{O A}=s_{i}$ as the original transmit symbol, and $\overrightarrow{O B}=\lambda_{i} s_{i}$ as the transmit symbol affected by the mutual coupling effect. Note that due to the existence of the angle rotation for exploiting the constructive region, each $\lambda_{i}$ is a complex variable and generally we have

$$
\Im\left(\lambda_{i}\right) \neq 0, \quad \forall i \in\left\{1,2, \ldots, N_{t}\right\} .
$$


Then, to have $\overrightarrow{O B}$ located in the constructive region, based on Fig. 2 the following condition should be satisfied:

$$
\theta_{A B}=\arctan \left(\frac{|\overrightarrow{B C}|}{|\overrightarrow{A C}|}\right) \leq \theta_{t},
$$

where for $\mathcal{M}$-PSK modulation, the threshold of the angle is given by

$$
\theta_{t}=\frac{\pi}{\mathcal{M}}
$$

Based on Fig. 2, the resulting symbol after mutual coupling can be expressed as

$$
\overrightarrow{O B}=\overrightarrow{O A}+\overrightarrow{A B}=s_{i}+\overrightarrow{A B}=\lambda_{i} s_{i}
$$

which leads to the expression of the interfering signal, given by

$$
\overrightarrow{A B}=\left(\lambda_{i}-1\right) s_{i}
$$

Noting that $\overrightarrow{A C}$ and $\overrightarrow{C B}$ are perpendicular, we could further obtain

$$
\overrightarrow{A C}=\left[\Re\left(\lambda_{i}\right)-1\right] s_{i},
$$

and

$$
\begin{aligned}
\overrightarrow{C B} & =\overrightarrow{A B}-\overrightarrow{A C}=\left(\lambda_{i}-1\right) s_{i}-\left[\Re\left(\lambda_{i}\right)-1\right] s_{i} \\
& =j \cdot \Im\left(\lambda_{i}\right) s_{i},
\end{aligned}
$$

where the imaginary unit $j$ denotes the angle rotation of $90^{\circ}$. It is then observed that the real part of each $\lambda_{i}$ represents the scaling of the original transmit symbol, and the imaginary part represents the angle rotation of the symbol. Based on (31), (26) can be transformed into

$$
\begin{aligned}
\theta_{A B} & =\arctan \left[\frac{\Im\left(\lambda_{i}\right)}{\Re\left(\lambda_{i}\right)-1}\right] \leq \theta_{t} \\
& \Rightarrow\left[\Re\left(\lambda_{i}\right)-1\right] \cdot \tan \left(\theta_{t}\right) \geq\left|\Im\left(\lambda_{i}\right)\right| .
\end{aligned}
$$

It is then shown in (32) that each $\lambda_{i}$ should satisfy $\Re\left(\lambda_{i}\right) \geq 1$ so that (32) can be met, which means that compared to the original transmit symbol $s_{i}$, the transmit symbol after mutual coupling will have a larger distance to the detection threshold, and therefore an improved detection performance can be expected.

Then, what is left is to determine each value of the load impedance $z_{L_{i}}$ so that phase alignment can be achieved. Recall (18) which forms the relationship between the load impedance $z_{L_{i}}$ and the scaling factor $\lambda_{i}$, which can then be transformed into

$$
\begin{aligned}
& \tilde{\boldsymbol{\Gamma}} \mathbf{Z}_{d}^{-1} \operatorname{diag}(\mathbf{\Lambda}) \mathbf{s}=[\mathbf{I}-\operatorname{diag}(\boldsymbol{\Lambda})] \mathbf{s} \\
& \quad \Rightarrow \tilde{\boldsymbol{\Gamma}} \operatorname{diag}\left(\lambda_{i} s_{i}\right) \operatorname{vec}\left(\mathbf{Z}_{d}^{-1}\right)=[\mathbf{I}-\operatorname{diag}(\mathbf{\Lambda})] \mathbf{s} \\
& \quad \Rightarrow \operatorname{vec}\left(\mathbf{Z}_{d}^{-1}\right)=\left[\tilde{\boldsymbol{\Gamma}} \operatorname{diag}\left(\lambda_{i} s_{i}\right)\right]^{-1}[\mathbf{I}-\operatorname{diag}(\mathbf{\Lambda})] \mathbf{s} \\
& \quad \Rightarrow \operatorname{vec}\left(\mathbf{Z}_{d}^{-1}\right)=\operatorname{diag}\left(\frac{1}{\lambda_{i} s_{i}}\right) \tilde{\boldsymbol{\Gamma}}^{-1}[\mathbf{I}-\operatorname{diag}(\mathbf{\Lambda})] \mathbf{s} .
\end{aligned}
$$

As can be seen, given a transmit symbol vector $\mathbf{s}$, we can always obtain vec $\left(\mathbf{z}_{d}^{-1}\right)$ and further $z_{L_{i}}$ with an arbitrary large scaling vector. However, an arbitrary scaling vector $\operatorname{diag}(\boldsymbol{\Lambda})$ may lead to impractical values for $z_{L_{i}}$, as the real part of $z_{L_{i}}$ should be positive such that the antenna array can radiate power [43], [44]. Therefore, we employ convex optimization to determine the scaling vector under realistic implementation constraints, where we consider two optimization criteria, as detailed below.

Max-Min: Noting that each $\Re\left(\lambda_{i}\right)$ represents the scaling of the original transmit symbol, we therefore consider a max-min optimization problem where we maximize the minimum value of $\Re\left(\lambda_{i}\right)$, formulated as

$$
\begin{aligned}
\mathcal{P}_{1}: \quad & \max _{\lambda_{i}, z_{L_{i}}} \Re\left(\lambda_{i}\right) \\
\text { s.t. } & \left(\lambda_{i}-1\right) s_{i}+\left[\tilde{\mathbf{\Gamma}} \mathbf{Z}_{d}^{-1} \operatorname{diag}(\mathbf{\Lambda}) \mathbf{s}\right]_{i}=0, \forall i \in I \\
& {\left[\Re\left(\lambda_{i}\right)-1\right] \cdot \tan \left(\theta_{t}\right) \geq\left|\Im\left(\lambda_{i}\right)\right|, \quad \forall i \in I } \\
& \Re\left(z_{L_{i}}\right) \geq 0, \quad \forall i \in I \\
& \Re\left(\lambda_{i}\right) \geq 1, \quad \forall i \in I
\end{aligned}
$$

where we denote $I=\left\{1,2, \ldots, N_{t}\right\}$ for simplicity. By using (12) and by denoting

$$
t_{i}=\lambda_{i}-1, x_{i}=\frac{\lambda_{i}}{z_{A}+z_{L_{i}}},
$$

and

$$
\mathbf{x}=\left[x_{1}, x_{2}, \ldots, x_{N_{t}}\right]^{T},
$$

the max-min problem in (34) can be further transformed into

$$
\begin{aligned}
\mathcal{P}_{2}: \quad \max _{x_{i}, t_{i}} \omega & \\
\text { s.t. } & t_{i} s_{i}+[\tilde{\mathbf{\Gamma}} \operatorname{diag}(\mathbf{x}) \mathbf{s}]_{i}=0, \quad \forall i \in I \\
& \Re\left(t_{i}\right) \cdot \tan \left(\theta_{t}\right) \geq\left|\Im\left(t_{i}\right)\right|, \quad \forall i \in I \\
& \Re\left(z_{L_{i}}\right) \geq 0, \quad \forall i \in I \\
& \omega \leq \Re\left(t_{i}\right)+1, \quad \forall i \in I
\end{aligned}
$$

where $\omega$ is an introduced auxiliary variable. Based on (35), we can express the load impedance $z_{L_{i}}$ as

$$
z_{L_{i}}=\frac{t_{i}+1}{x_{i}}-z_{A} \text {. }
$$

Then, as each $t_{i}$ is a complex variable, the constraint that $\Re\left(z_{L_{i}}\right) \geq 0$ is equivalent to

$$
\begin{aligned}
& \Re\left(\frac{t_{i}+1}{x_{i}}\right) \geq \Re\left(z_{A}\right) \\
& \quad \Rightarrow \frac{\left[\Re\left(t_{i}\right)+1\right] \Re\left(x_{i}\right)+\Im\left(t_{i}\right) \Im\left(x_{i}\right)}{\left|x_{i}\right|^{2}} \geq \Re\left(z_{A}\right) \\
& \quad \Rightarrow\left[\Re\left(t_{i}\right)+1\right] \Re\left(x_{i}\right)+\Im\left(t_{i}\right) \Im\left(x_{i}\right) \geq \Re\left(z_{A}\right) \cdot\left|x_{i}\right|^{2} .
\end{aligned}
$$

Note that (39) is a complicated non-convex constraint that makes the optimization non-convex, a relaxation is needed to transform this constraint into a convex one. In this paper, this constraint is relaxed in the following way: by adding a parameter $\mu_{t h}$ multiplied to the right-hand side of the constraint, 
we remove the imaginary part multiplication $\mathfrak{I}\left(t_{i}\right) \mathfrak{I}\left(x_{i}\right)$ at the left-hand side, and (39) is transformed into

$$
\left[\Re\left(t_{i}\right)+1\right] \Re\left(x_{i}\right) \geq \mu_{t h} \Re\left(z_{A}\right) \cdot\left|x_{i}\right|^{2},
$$

where $\mu_{t h} \geq 1$. With a larger value of $\mu_{t h}$, there is a higher possibility that the optimization problem is solved with a practical solution. However, a larger value of $\mu_{t h}$ will also lead to a loss in the performance gains as it reduces the value range of the variables, which will be numerically studied in the simulation results. Then, we further impose a relatively strong constraint to relax the non-convex constraint (40) such that the solution obtained with the relaxed constraint also satisfies the original constraint. With $\left[\Re\left(t_{i}\right)+1\right] \geq 1$, we have

$$
\Re\left(z_{A}\right) \cdot\left|x_{i}\right|^{2} \geq \frac{\Re\left(z_{A}\right)}{\Re\left(t_{i}\right)+1} \cdot\left|x_{i}\right|^{2} .
$$

Then, if the following constraint is satisfied

$$
\Re\left(x_{i}\right) \geq \mu_{t h} \Re\left(z_{A}\right) \cdot\left|x_{i}\right|^{2},
$$

the constraint in (40) is also satisfied. In this way, the nonconvex constraint (39) has been substituted with a convex constraint as shown in (42), and the final optimization problem is formulated as

$$
\begin{aligned}
\mathcal{P}_{3}: \quad \max _{x_{i}, t_{i}} \omega & \\
\text { s.t. } & t_{i} s_{i}+[\tilde{\boldsymbol{\Gamma}} \operatorname{diag}(\mathbf{x}) \mathbf{s}]_{i}=0, \quad \forall i \in I \\
& \Re\left(t_{i}\right) \cdot \tan \left(\theta_{t}\right) \geq\left|\widetilde{\Im}\left(t_{i}\right)\right|, \quad \forall i \in I \\
& \Re\left(x_{i}\right) \geq \mu_{t h} \Re\left(z_{A}\right) \cdot\left|x_{i}\right|^{2}, \quad \forall i \in I \\
& \omega \leq \Re\left(t_{i}\right)+1, \quad \forall i \in I
\end{aligned}
$$

which is a second-order cone programming (SOCP) and can be efficiently solved by convex optimization tools such as CVX or SeDuMi.

Sum-Max: in addition to the max-min optimization problem, we also pursue an optimization problem that targets at maximizing the average performance improvement for the data symbol. In this case, a sum-max problem can also be formulated where we maximize the summation of the scaling factor $\Re\left(\lambda_{i}\right)$ divided by $N_{t}$, expressed as

$$
\begin{aligned}
\mathcal{P}_{4}: \quad \max _{\lambda_{i}, z_{L_{i}}} & \frac{\sum_{i=1}^{N_{t}} \Re\left(\lambda_{i}\right)}{N_{t}} \\
\text { s.t. } & \left(\lambda_{i}-1\right) s_{i}+\left[\tilde{\boldsymbol{\Gamma}} \mathbf{Z}_{d}^{-1} \operatorname{diag}(\mathbf{\Lambda}) \mathbf{s}\right]_{i}=0, \quad \forall i \in I \\
& \Re\left(t_{i}\right) \cdot \tan \left(\theta_{t}\right) \geq\left|\Im\left(\lambda_{i}\right)\right|, \quad \forall i \in I \\
& \Re\left(z_{L_{i}}\right) \geq 0, \quad \forall i \in I \\
& \Re\left(\lambda_{i}\right) \geq 1, \quad \forall i \in I
\end{aligned}
$$

which can be similarly transformed into a convex version, obtained as

$$
\begin{aligned}
\mathcal{P}_{5}: \quad \max _{x_{i}, t_{i}} & \sum_{i=1}^{N_{t}} \Re\left(t_{i}\right) \\
\text { s.t. } & t_{i} s_{i}+[\tilde{\boldsymbol{\Gamma}} \operatorname{diag}(\mathbf{x}) \mathbf{s}]_{i}=0, \quad \forall i \in I \\
& \Re\left(t_{i}\right) \cdot \tan \left(\theta_{t}\right) \geq \mid \widetilde{\Im}\left(t_{i}\right), \quad \forall i \in I \\
& \Re\left(x_{i}\right) \geq \mu_{t h} \Re\left(z_{A}\right) \cdot\left|x_{i}\right|^{2}, \quad \forall i \in I
\end{aligned}
$$

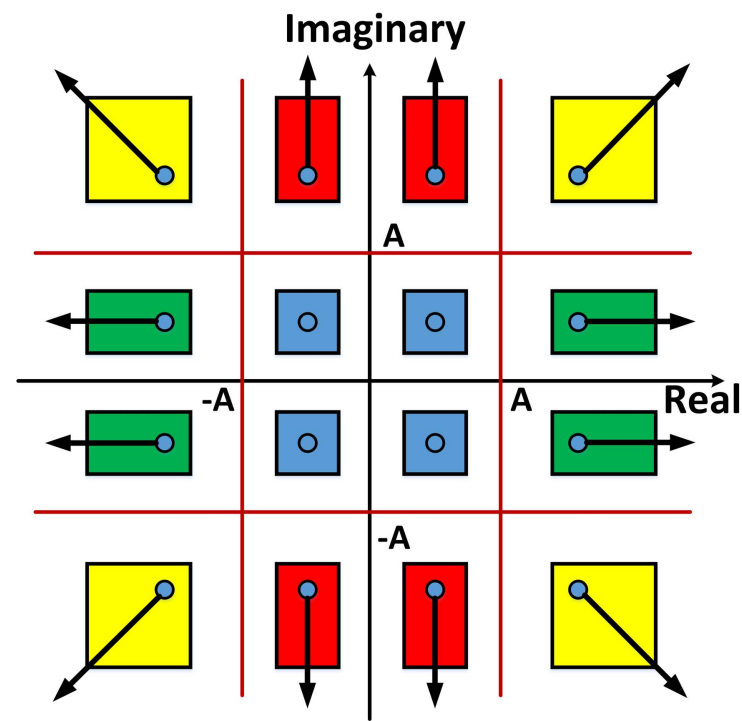

Fig. 3. Normalized 16QAM constellation points and the corresponding constructive region.

After the optimization problem is solved, the load impedance for each antenna can be calculated based on (38).

\section{Exploiting Mutual Coupling for QAM Modulations}

The proposed schemes in the previous section assume PSK modulations, and in this section we extend the proposed schemes to QAM modulations. For QAM modulations, we propose to introduce the scaling factor $\lambda_{i}^{R E}$ and $\lambda_{i}^{I M}, \forall i \in I$ for the real and imaginary part of the transmit symbol $s_{i}$, respectively. Then, for QAM modulations (16) can be expressed as

$$
\mathbf{Z}_{d}\left(\mathbf{Z}_{d}+\tilde{\boldsymbol{\Gamma}}\right)^{-1} \mathbf{s}=\operatorname{diag}\left(\boldsymbol{\Lambda}_{R E}\right) \Re(\mathbf{s})+j \cdot \operatorname{diag}\left(\boldsymbol{\Lambda}_{I M}\right) \Im(\mathbf{s}),
$$

where

$$
\begin{aligned}
& \boldsymbol{\Lambda}_{R E}=\left[\lambda_{1}^{R E}, \lambda_{2}^{R E}, \ldots, \lambda_{N_{t}}^{R E}\right]^{T}, \\
& \boldsymbol{\Lambda}_{I M}=\left[\lambda_{1}^{I M}, \lambda_{2}^{I M}, \ldots, \lambda_{N_{t}}^{I M}\right]^{T} .
\end{aligned}
$$

Then, (46) can be further transformed into

$$
\mathbf{s}=\left(\mathbf{Z}_{d}+\tilde{\boldsymbol{\Gamma}}\right) \mathbf{Z}_{d}^{-1}\left[\operatorname{diag}\left(\boldsymbol{\Lambda}_{R E}\right) \Re(\mathbf{s})+j \cdot \operatorname{diag}\left(\boldsymbol{\Lambda}_{I M}\right) \Im(\mathbf{s})\right],
$$

which is equivalent to

$$
\begin{aligned}
& {\left[\operatorname{diag}\left(\boldsymbol{\Lambda}_{R E}-\mathbf{I}\right) \Re(\mathbf{s})+j \cdot \operatorname{diag}\left(\boldsymbol{\Lambda}_{I M}-\mathbf{I}\right) \Im(\mathbf{s})\right]} \\
& \quad+\tilde{\mathbf{\Gamma}} \mathbf{Z}_{d}^{-1}\left[\operatorname{diag}\left(\boldsymbol{\Lambda}_{R E}\right) \Re(\mathbf{s})+j \cdot \operatorname{diag}\left(\boldsymbol{\Lambda}_{I M}\right) \Im(\mathbf{s})\right]=\mathbf{0} .
\end{aligned}
$$

We then proceed to consider the constructive interference constraints. For QAM modulations, the constructive interference can be exploited for the outer constellation points. To demonstrate this, we employ 16QAM as the example and in Fig. 3 the normalized constellation points and the corresponding constructive region for 16QAM are shown, where $A=0.6324$ 


$$
\begin{aligned}
\mathcal{P}_{6}: \quad \max _{\lambda_{i}^{U}, z_{L_{i}}} \min \left(\lambda_{i}^{U}\right) & \\
\text { s.t. } & {\left[\left(\lambda_{i}^{R E}-1\right) \Re\left(s_{i}\right)+j \cdot\left(\lambda_{i}^{I M}-1\right) \Im\left(s_{i}\right)\right]+\left\{\tilde{\mathbf{\Gamma}} \mathbf{Z}_{d}^{-1}\left[\operatorname{diag}\left(\mathbf{\Lambda}_{R E}\right) \Re(\mathbf{s})+j \cdot \operatorname{diag}\left(\boldsymbol{\Lambda}_{I M}\right) \Im(\mathbf{s})\right]\right\}_{i}=0, \forall i \in I } \\
& \Re\left(z_{L_{i}}\right) \geq 0, \forall i \in I \\
& \lambda_{i}^{R E} \geq 1, \lambda_{i}^{I M} \geq 1 \text { if }\left|\Re\left(s_{i}\right)\right| \geq A \text { and }\left|\Im\left(s_{i}\right)\right| \geq A, \forall i \in I \\
& \lambda_{i}^{R E}=1, \lambda_{i}^{I M} \geq 1 \text { if }\left|\Re\left(s_{i}\right)\right| \leq A \text { and }\left|\Im\left(s_{i}\right)\right| \geq A, \forall i \in I \\
& \lambda_{i}^{R E} \geq 1, \lambda_{i}^{I M}=1 \text { if }\left|\Re\left(s_{i}\right)\right| \geq A \text { and }\left|\Im\left(s_{i}\right)\right| \leq A, \forall i \in I \\
& \lambda_{i}^{R E}=1, \lambda_{i}^{I M}=1 \text { if }\left|\Re\left(s_{i}\right)\right| \leq A \text { and }\left|\Im\left(s_{i}\right)\right| \leq A, \forall i \in I \\
& \mathcal{U} \in\{R E, I M\}
\end{aligned}
$$

denotes the detection threshold for the normalized constellation. Then, for the exploitation of the constructive interference, the constellation points can be divided into 4 types:

1) Type 1 (denoted as yellow in Fig. 3): $\left|\Re\left(s_{i}\right)\right| \geq A$ and $\left|\Im\left(s_{i}\right)\right| \geq A$. In this case, the phase alignment is similar to PSK, and to achieve the constructive interference we have $\lambda_{i}^{R E} \geq 1$ and $\lambda_{i}^{I M} \geq 1$

2) Type 2 (denoted as red in Fig. 3): $\left|\Re\left(s_{i}\right)\right| \leq A$ and

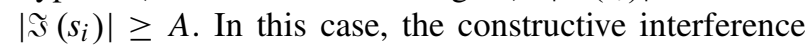
can only be exploited for the imaginary part of each transmit symbol, and we can then obtain $\lambda_{i}^{R E}=1$ and $\lambda_{i}^{I M} \geq 1$

3) Type 3 (denoted as green in Fig. 3): $\left|\Re\left(s_{i}\right)\right| \geq A$ and $\left|\mathfrak{s}\left(s_{i}\right)\right| \leq A$. In this case, the constructive interference can only be exploited for the real part of each transmit symbol, and we can then obtain $\lambda_{i}^{R E} \geq 1$ and $\lambda_{i}^{I M}=1$;

4) Type 4 (denoted as blue in Fig. 3): $\left|\Re\left(s_{i}\right)\right| \leq A$ and $\left|\mathfrak{\Im}\left(s_{i}\right)\right| \leq A$. In this case, any interference is destructive, and therefore we have $\lambda_{i}^{R E}=1$ and $\lambda_{i}^{I M}=1$.

Based on the above analysis, we can then formulate the max-min optimization problem for 16QAM in (50), which is shown on the top of this page. Then, by denoting

$$
t_{i}^{R E}=\lambda_{i}^{R E}-1, t_{i}^{I M}=\lambda_{i}^{I M}-1, \forall i \in I,
$$

and

$$
\mathbf{x}=\left[x_{1}, x_{2}, \ldots, x_{N_{t}}\right]^{T},
$$

where each $x_{i}$ is defined as

$$
x_{i}=\frac{\lambda_{i}^{R E} \Re\left(s_{i}\right)+j \cdot \lambda_{i}^{I M} \Im\left(s_{i}\right)}{z_{A}+z_{L_{i}}},
$$

the constraint that $\Re\left(z_{L_{i}}\right) \geq 0$ can be further transformed into

$$
\Re\left[\frac{\lambda_{i}^{R E} \Re\left(s_{i}\right)+j \cdot \lambda_{i}^{I M} \Im\left(s_{i}\right)}{x_{i}}\right] \geq \Re\left(z_{A}\right),
$$

which is equivalent to

$$
\frac{\left(t_{i}^{R E}+1\right) \Re\left(s_{i}\right) \Re\left(x_{i}\right)+\left(t_{i}^{I M}+1\right) \Im\left(s_{i}\right) \Im\left(x_{i}\right)}{\left|x_{i}\right|^{2}} \geq \Re\left(z_{A}\right) .
$$

By following a similar relaxation approach in (40)-(42), this constraint can be relaxed into a convex form as

$$
\Re\left(s_{i}\right) \Re\left(x_{i}\right)+\Im\left(s_{i}\right) \Im\left(x_{i}\right) \geq\left|x_{i}\right|^{2} \cdot \Re\left(z_{A}\right)
$$

Then, we can formulate the final optimization problem for 16QAM as

$\mathcal{P}_{7}$ :

$$
\begin{array}{ll}
\max _{x_{i}, t_{i}^{U}} & \omega \\
\text { s.t. } & {\left[t_{i}^{R E} \Re\left(s_{i}\right)+j \cdot t_{i}^{I M} \Im\left(s_{i}\right)\right]+[\tilde{\boldsymbol{\Gamma}} \mathbf{x}]_{i}=0} \\
& \Re\left(s_{i}\right) \Re\left(x_{i}\right)+\Im\left(s_{i}\right) \Im\left(x_{i}\right) \geq\left|x_{i}\right|^{2} \cdot \Re\left(z_{A}\right) \\
& \lambda_{i}^{R E} \geq 1, \lambda_{i}^{I M} \geq 1 \text { if }\left|\Re\left(s_{i}\right)\right| \geq A \text { and }\left|\Im\left(s_{i}\right)\right| \geq A \\
& \lambda_{i}^{R E}=1, \lambda_{i}^{I M} \geq 1 \text { if }\left|\Re\left(s_{i}\right)\right| \leq A \text { and }\left|\Im\left(s_{i}\right)\right| \geq A \\
& \lambda_{i}^{R E} \geq 1, \lambda_{i}^{I M}=1 \text { if }\left|\Re\left(s_{i}\right)\right| \geq A \text { and }\left|\Im\left(s_{i}\right)\right| \leq A \\
& \lambda_{i}^{R E}=1, \lambda_{i}^{I M}=1 \text { if }\left|\Re\left(s_{i}\right)\right| \leq A \text { and }\left|\Im\left(s_{i}\right)\right| \leq A \\
& \omega \leq \Re\left(t_{i}^{u}\right)+1 \\
& \mathcal{U} \in\{R E, I M\} \\
& \forall i \in I
\end{array}
$$

The optimization problem $\mathcal{P}_{7}$ can then be efficiently solved with convex optimization tools, and each optimal load impedance can then be obtained based on (53), given by

$$
z_{L_{i}}=\frac{\lambda_{i}^{R E} \Re\left(s_{i}\right)+j \cdot \lambda_{i}^{I M} \Im\left(s_{i}\right)}{x_{i}}-z_{A} .
$$

The formulation of the sum-max problem can be obtained in a similar way and is omitted for brevity.

\section{Performance Analysis}

In this section, the performance analysis of the proposed schemes is conducted, where we focus on the zero-forcing receivers. The analysis of the correlated channel model employed in this paper is firstly presented, followed by the analytical probability of error.

\section{A. Correlated Channel Characterization}

We firstly study the property of the correlated channels introduced in Section II. Based on (5) and note that $\mathbf{H}_{\alpha}$ is diagonal, we can express the elements in $\mathbf{H}$ as

$$
\mathbf{H}(i, j)=\frac{1}{\sqrt{M}} \sum_{m=1}^{M} \mathbf{A}_{r}(i, m) \mathbf{A}_{t}^{H}(m, j) \cdot \alpha_{m} .
$$

Noting that $\alpha_{m} \sim \mathcal{C} N(0,1)$ and each $\alpha_{m}$ is independent, therefore each element in $\mathbf{H}$ is the summation of independent 
Gaussian variables, which follows a Gaussian distribution with zero mean. The resulting channel matrix $\mathbf{H}$ is therefore also Gaussian with zero mean. We then proceed to derive the covariance matrix of $\mathbf{H}$. For ease of analysis, we only consider the expectation over the Rayleigh components and regard $\mathbf{A}_{r}$ and $\mathbf{A}_{t}$ as constant matrices throughout the derivation. Then, based on the definition, the covariance matrix can be calculated as

$$
\begin{aligned}
\mathbf{C}_{\mathbf{H}} & =\mathbb{E}\left\{[\mathbf{H}-\mathbb{E}(\mathbf{H})] \cdot[\mathbf{H}-\mathbb{E}(\mathbf{H})]^{H}\right\} \\
& =\mathbb{E}\left\{\left(\mathbf{A}_{r} \mathbf{H}_{\alpha} \mathbf{A}_{t}^{H}\right) \cdot\left(\mathbf{A}_{r} \mathbf{H}_{\alpha} \mathbf{A}_{t}^{H}\right)^{H}\right\} \\
& =\mathbb{E}\left\{\mathbf{A}_{r} \mathbf{H}_{\alpha} \mathbf{A}_{t}^{H} \mathbf{A}_{t} \mathbf{H}_{\alpha}^{H} \mathbf{A}_{r}^{H}\right\} \\
& =\mathbf{A}_{r} \cdot \mathbb{E}\left\{\mathbf{H}_{\alpha} \mathbf{A}_{t}^{H} \mathbf{A}_{t} \mathbf{H}_{\alpha}^{H}\right\} \cdot \mathbf{A}_{r}^{H} \\
& =\mathbf{A}_{r} \cdot \mathbb{E}\{\mathbf{T}\} \cdot \mathbf{A}_{r}^{H}
\end{aligned}
$$

where for simplicity we denote

$$
\begin{aligned}
\mathbf{T} & =\mathbf{H}_{\alpha} \mathbf{A}_{t}^{H} \mathbf{A}_{t} \mathbf{H}_{\alpha}^{H} \\
& =\mathbf{H}_{\alpha} \mathbf{A} \mathbf{H}_{\alpha}^{H},
\end{aligned}
$$

where $\mathbf{A}=\mathbf{A}_{t}^{H} \mathbf{A}_{t}$. Note that $\mathbf{H}_{\alpha}$ is diagonal, and each element in $\mathbf{T}$ can then be calculated as

$$
\mathbf{T}(i, j)=\frac{1}{M} \alpha_{i} \cdot \mathbf{A}(i, j) \cdot \alpha_{j}^{H} .
$$

Based on Section II, each element in $\mathbf{H}_{\alpha}$ is independent and follows a standard normal distribution, and therefore we have

$$
\begin{aligned}
& \forall i \neq j, \mathbb{E}\left\{\alpha_{i} \alpha_{j}^{H}\right\}=0, \\
& \mathbb{E}\left\{\alpha_{i} \alpha_{i}^{H}\right\}=1,
\end{aligned}
$$

which further leads to

$$
\begin{aligned}
& \forall i \neq j, \mathbb{E}\{\mathbf{T}(i, j)\}=0, \\
& \mathbb{E}\{\mathbf{T}(i, i)\}=\frac{1}{M} \cdot \mathbf{A}_{\text {diag }},
\end{aligned}
$$

where $\mathbf{A}_{\text {diag }}=\operatorname{diag}[\mathbf{A}(1,1), \mathbf{A}(2,2), \ldots, \mathbf{A}(M, M)]$ is a diagonal matrix with diagonal elements being the diagonal elements of A. Finally, based on the above derivation, the covariance matrix of $\mathbf{H}$ can be obtained as

$$
\mathbf{C}_{\mathbf{H}}=\frac{1}{M} \cdot \mathbf{A}_{r} \mathbf{A}_{\text {diag }} \mathbf{A}_{r}^{H} .
$$

\section{B. Probability of Error}

Based on the analysis on the correlated channel models, we can now study the analytical probability of error for the proposed schemes. For conventional ZF receiver, the analytical bit error rate (BER) over Rayleigh fading channels for QPSK, as assumed in this paper, can be obtained as [51]

$$
\begin{aligned}
P_{e}= & \sum_{k=1}^{N_{t}} \frac{1}{2}-\sqrt{\frac{\gamma_{k}}{\pi}} \cdot \frac{\Gamma\left(N_{r}-N_{t}+\frac{3}{2}\right)}{\Gamma\left(N_{r}-N_{t}+1\right)} \\
& \times_{2} F_{1}\left(\left[\frac{1}{2}, N_{r}-N_{t}+\frac{3}{2}\right] ; \frac{3}{2} ;-\gamma_{k}\right),
\end{aligned}
$$

where $\Gamma(\cdot)$ denotes the Gamma function, and ${ }_{2} F_{1}([a, b] ; c ; z)$ is the hypergeometric function [52]. $\gamma_{k}$ can be obtained based on the signal-to-noise ratio per bit $\left(E_{b} N_{0}\right)$ and the covariance matrix of the correlated channels, which is expressed as

$$
\gamma_{k}=\frac{1}{\sigma^{2} \mathbf{C}_{\mathbf{H}}^{-1}(k, k)},
$$

where $\mathbf{C}_{\mathbf{H}}^{-1}(k, k)$ is the $k$-th diagonal element of the inverse matrix of the covariance matrix $\mathbf{C}_{\mathbf{H}}$.

For the proposed scheme, we propose to exploit the mutual coupling effect to benefit the system performance. With phase alignment achieved, each transmit symbol $s_{i}$ is enhanced by a factor $\lambda_{i}$, which can be translated as an increase in the transmit power, and $\left|\lambda_{i}\right|^{2}$ can be regarded as the power improvement factor, without incurring additional interference. Therefore, the analytical BER for the proposed schemes based on ZF receiver can also be obtained by (66), where $\hat{\gamma}_{k}$ for the proposed scheme is obtained as

$$
\hat{\gamma}_{k}=\frac{\left|\lambda_{i}\right|^{2}}{\sigma^{2} \mathbf{C}_{\mathbf{H}}^{-1}(k, k)} .
$$

\section{PRACTICAL IMPLEMENTATION}

In this section, the implementation of the proposed schemes for practical application is explored, where a number of practical implementation issues are discussed.

\section{A. Extraction of $\mathbf{H}$}

From (3) we observe the receiver needs to obtain $\mathbf{H}$ to perform the equalization, while with pilots we can only obtain $\mathbf{H Z}$. Therefore, we need to extract $\mathbf{H}$ from $\mathbf{H Z}$. We note that the mutual impedance matrix $\boldsymbol{\Gamma}$ is only dependent on the array structure and does not change, and then typically $\boldsymbol{\Gamma}$ can be known to the transmitter either by the induced EMF method or other experimental measurements such as boundary-value approach and transmission-line method [25]. At the pilot stage, the channel is obtained by firstly setting each load impedance to a specific reference value, such as $z_{L_{i}}=50 \Omega$, $\forall i \in\left\{1,2, \ldots, N_{t}\right\}$, which is known to both the transmitter and the receiver. We then denote the resulting load impedance vector as $\mathbf{z}_{L}^{0}$ and mutual coupling matrix as $\mathbf{Z}_{0}$. With pilots we can obtain $\mathbf{H} \mathbf{Z}_{0}$, and as $\mathbf{Z}_{0}$ is known to the receiver, $\mathbf{H}$ can be extracted from $\mathbf{H Z}_{0}$ to obtain the equalizer $\mathbf{G}$, and the proposed schemes can then be applied during data transmission.

\section{B. Use of Lookup Tables}

Based on (18) and the description of the proposed techniques, it can be observed that the optimal value of load impedance for each antenna element is solely dependent on the transmit symbol vector, irrespective of the channel matrix, which therefore enables the design of a lookup table for the optimal values of load impedance based on the transmit symbol vector. With this approach, the optimization process can be conducted off-line before transmission to obtain the optimal values of the load impedances for each possible transmit symbol combination. This information can then be 
kept and used for future data transmission, where a symbolby-symbol optimization is therefore no longer needed, which can significantly reduce the computational complexity. If we assume $\mathcal{M}$-PSK modulation is employed, then the total number of possible combinations for the transmit symbol vector will be $L=\mathcal{M}^{N_{t}}$, which is equal to the length of the lookup table. To illustrate this, an example of the lookup table is given where we assume QPSK modulation is employed with 2 transmit antennas, which leads to a total number of 16 possible combinations of the transmit vector. For QPSK, without loss of generality we denote $m_{1}=(1,1)$, $m_{2}=(1,-1), m_{3}=(-1,-1)$, and $m_{4}=(-1,1)$. The lookup table is then obtained and given in Table. I, where the optimization is based on $\mathcal{P}_{3}$.

\section{Solutions for Extreme Load Impedance Values}

It should be noted that, for some transmit symbol vector, the solutions obtained by convex optimization lead to large negative values of load impedances, which is unreasonable and means that there exist no practical solutions for such symbol combinations. In this example, this happens when the two antennas transmit the same symbols, which are $\left[m_{1}, m_{1}\right]^{T}$, $\left[m_{2}, m_{2}\right]^{T},\left[m_{3}, m_{3}\right]^{T}$ and $\left[m_{4}, m_{4}\right]^{T}$. This can be explained by taking a look at the constraint in (18), which can be expressed as

$$
\left[(\operatorname{diag}(\mathbf{\Lambda})-\mathbf{I})+\tilde{\mathbf{\Gamma}} \mathbf{Z}_{d}^{-1} \operatorname{diag}(\mathbf{\Lambda})\right] \mathbf{s}=\mathbf{0} .
$$

When the same symbols are transmitted at each antenna element, (69) is equivalent to

$$
\begin{aligned}
& \operatorname{diag}(\mathbf{\Lambda})+\tilde{\mathbf{\Gamma}} \mathbf{Z}_{d}^{-1} \operatorname{diag}(\mathbf{\Lambda})=\mathbf{I} \\
& \quad \Rightarrow\left(\mathbf{I}+\tilde{\mathbf{\Gamma}} \mathbf{Z}_{d}^{-1}\right) \operatorname{diag}(\boldsymbol{\Lambda})=\mathbf{I} .
\end{aligned}
$$

As diag ( $\mathbf{\Lambda})$ is a diagonal matrix, (70) can only be satisfied if $\left(\mathbf{I}+\tilde{\boldsymbol{\Gamma}} \mathbf{Z}_{d}^{-1}\right)$ is diagonal. However, $\tilde{\boldsymbol{\Gamma}}$ is a fixed matrix given in (13) with non-zero values for non-diagonal elements, and $\mathbf{Z}_{d}^{-1}$ is also not a zero matrix based on (12). Therefore, (70) cannot be satisfied for any value of $\lambda_{i}$ if all the transmit antennas transmit the same symbols, which explains the unreasonable solutions obtained from the convex optimization. Intuitively, this phenomenon is extended to larger transmit antenna numbers, where no practical solutions could be obtained when all the antennas transmit the same symbols. In this case, the fixed mutual coupling matrix is used where the value for all the load impedance is $z_{L_{i}}=50 \Omega$, $\forall i \in\left\{1,2, \ldots, N_{t}\right\}$, as shown in Table I. Furthermore, based on the above analysis, the feasibility probability of the proposed scheme can therefore be obtained as

$$
P_{\text {feasibility }}=1-\frac{\mathcal{M}}{\mathcal{M}^{N_{t}}}=1-\frac{1}{\mathcal{M}^{N_{t}-1}}
$$

Note that for the proposed schemes based on the constructive region of PSK modulations, the value of $\mu_{t h}$ also has an impact on the feasibility, and therefore $P_{\text {feasibility }}$ can be regarded as an upper bound. The effect of $\mu_{t h}$ on the feasibility probability is then studied numerically in the simulation results.
TABLE I

LOOKUP TABLE FOR $2 \times 2$ MIMO, QPSK, $P_{3}, d_{t}=0.2, d_{r}=0.5, d_{l}=0.3, \mu_{t h}=1.1$

\begin{tabular}{|c|c|c|c|}
\hline \multicolumn{2}{|c|}{ Transmit Symbol Vector } & \multicolumn{2}{c|}{ Optimal Impedance Values $(\Omega)$} \\
\hline Antenna 1 & Antenna 2 & Antenna 1 & Antenna 2 \\
\hline$m_{1}$ & $m_{1}$ & 50 & 50 \\
\hline$m_{1}$ & $m_{2}$ & $43.546-40.203 j$ & $52.009+26.114 j$ \\
\hline$m_{1}$ & $m_{3}$ & $45.812-7.396 j$ & $45.812-7.396 j$ \\
\hline$m_{1}$ & $m_{4}$ & $52.053+26.096 j$ & $43.532-40.181 j$ \\
\hline$m_{2}$ & $m_{1}$ & $49.217+27.758 j$ & $44.265-41.923 j$ \\
\hline$m_{2}$ & $m_{2}$ & 50 & 50 \\
\hline$m_{2}$ & $m_{3}$ & $43.567-40.277 j$ & $51.902+26.201 j$ \\
\hline$m_{2}$ & $m_{4}$ & $45.812-7.396 j$ & $45.812-7.396 j$ \\
\hline$m_{3}$ & $m_{1}$ & $45.812-7.396 j$ & $45.812-7.396 j$ \\
\hline$m_{3}$ & $m_{2}$ & $52.053+26.096 j$ & $43.532-40.181 j$ \\
\hline$m_{3}$ & $m_{3}$ & 50 & 50 \\
\hline$m_{3}$ & $m_{4}$ & $43.546-40.203 j$ & $52.009+26.114 j$ \\
\hline$m_{4}$ & $m_{1}$ & $43.567-40.277 j$ & $51.902+26.201 j$ \\
\hline$m_{4}$ & $m_{2}$ & $45.812-7.396 j$ & $45.812-7.396 j$ \\
\hline$m_{4}$ & $m_{3}$ & $49.217+27.758 j$ & $44.265-41.923 j$ \\
\hline$m_{4}$ & $m_{4}$ & 50 & 50 \\
\hline
\end{tabular}

\section{Symbol-by-Symbol Switching of Load Impedance Values}

Note that the proposed optimizations necessitate the adaptation of the load impedance values $z_{L_{i}}$ on a symbol-by-symbol basis, where for each data transmission, the value of each load impedance is tuned based on the lookup table to enable the application of the proposed schemes. It has been shown in [53] and the references therein that varactor technologies that support adaptive impedance tuning can be divided into 3 categories: semiconductor-based varactor diodes, microelectromechanical system (MEMS) varactors, and ferroelectric-based varactors. Specifically, semiconductor-based varactor diodes and ferroelectric-based varactors can support the tuning speed as fast as 1-100 ns [54]. Semiconductor-based varactor diodes are more attractive in low power design, while ferroelectricbased varactors have the capability for high power design. The tuning of the load impedance can be realized by a matching system based on an automated impedance tuning unit with ferroelectric varactors. Furthermore, recent studies on electronically steerable parasitic array radiators (ESPARs) where the radiation patterns are formed by tuning the load impedance for each parasitic element have shown that the frequent tuning of the load impedance is achievable [55]-[60]. This is verified by the proof-of-concept experiments in [61], which enables the implementation of the proposed schemes.

\section{Numerical Results}

In this section we evaluate the usefulness of the proposed schemes based on Monte Carlo simulations. We assume the system is operating at the frequency of $f=2.6 \mathrm{GHz}$, and the simulated channels are based on (5)-(8), where we assume $M=50$. A dipole antenna array is assumed, where the normalized antenna spacing at the transmitter and receiver is assumed as $d_{t}=0.2$ and $d_{r}=0.5$, respectively, and the dipole length of each antenna is $d_{l}=0.3$. The number of transmit and receive antennas is assumed as $N_{t}=4$ and $N_{r}=4$ respectively, while it is intuitive that the benefits of the proposed approaches extend to larger scale MIMO systems. Note that the proposed schemes are not dependent on the 
TABLE II

AbBREVIATIONS OF DifFERENT SCHEMES

\begin{tabular}{|c|c|}
\hline Abbreviations & Schemes \\
\hline ZF no MC (reference) & ZF equalizer with no mutual coupling \\
\hline ZF with MC & ZF equalizer with fixed mutual coupling \\
\hline max-min & the proposed scheme based on max-min $\mathcal{P}_{3}$ \\
\hline sum-max & the proposed scheme based on sum-max $\mathcal{P}_{5}$ \\
\hline
\end{tabular}

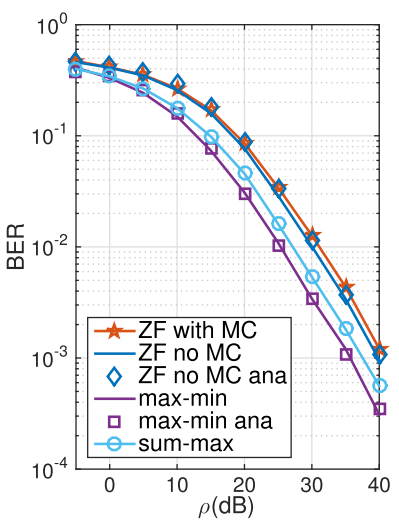

(a) BPSK

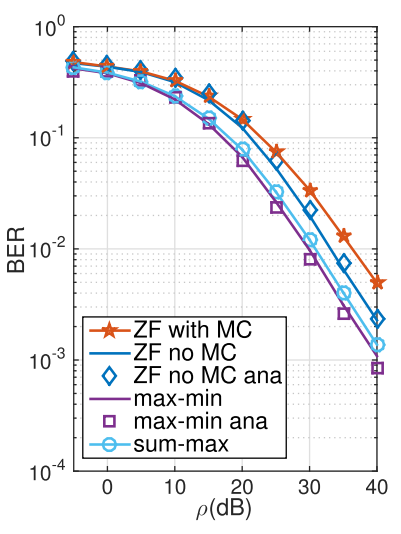

(b) QPSK
Fig. 4. BER performance of the proposed schemes, $4 \times 4, d_{t}=0.2$, $d_{r}=0.5, d_{l}=0.3, \mu_{t h}=1.1$

receiver structure, and for simplicity we assume $\mathrm{ZF}$ receiver is applied throughout the simulations, while the performance benefits intuitively extend to other receiver structures. The above parameters remain the same throughout the simulations, unless otherwise stated. For clarity, the abbreviations are summarized below in Table II.

In Fig. 4, the bit error rate (BER) performance of the proposed schemes for BPSK and QPSK is shown with respect to the transmit SNR. For both modulations, it is observed that "ZF with MC" is inferior to "ZF no MC" and achieves the worst BER performance, which is due to the fixed mutual coupling effect among antenna elements. For the proposed schemes, it can be observed that all the schemes outperform "ZF no MC" and "ZF with MC" with an SNR gain over $5 \mathrm{~dB}$ for QPSK, as the mutual coupling effect is exploited by the proposed techniques to improve the performance. Among all the proposed schemes, it can be seen that 'maxmin' generally outperforms 'sum-max' and achieves the best BER performance. While the proposed schemes require each antenna element equipped with a tunable load, the resulting performance gain is large enough and is worthy to pursue. As for the analytical results, a close match can be observed for conventional "ZF no MC" and the proposed schemes.

In Fig. 5, the throughput benefits of the proposed schemes are shown, where the throughput is defined as

$$
\begin{aligned}
T_{r} & =(1-\mathrm{BLER}) \cdot m \cdot N_{t} \text { bits/channel use } \\
& =\left(1-P_{b}\right)^{F \cdot m} \cdot m \cdot N_{t} \text { bits } / \text { channel use. }
\end{aligned}
$$

In (72), BLER denotes the block error rate, and $P_{b}$ is the BER. $m=2$ bits/symbol for QPSK and $m=1 \mathrm{bit} / \mathrm{symbol}$ for BPSK. The block length used for the simulations

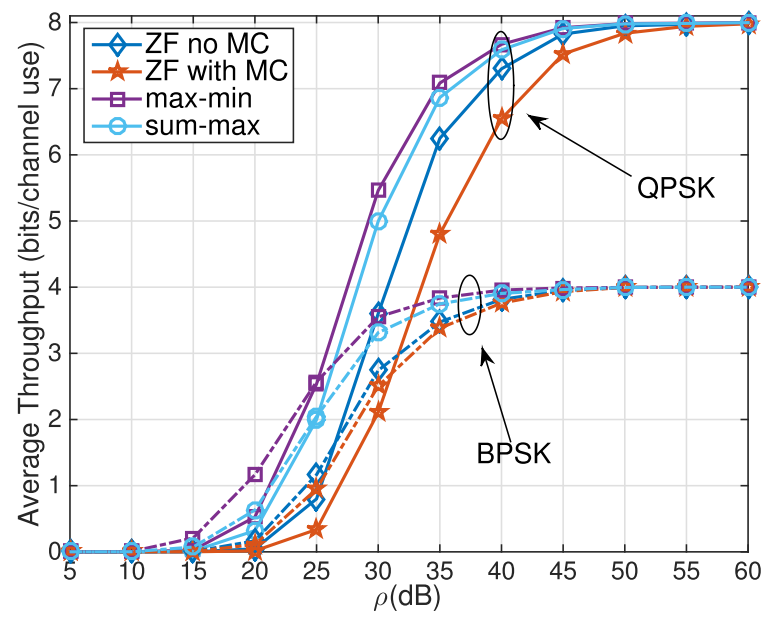

Fig. 5. Average throughput of different schemes, $4 \times 4, d_{t}=0.2, d_{r}=0.5$ $d_{l}=0.3, \mu_{t h}=1.1$, BPSK and QPSK.

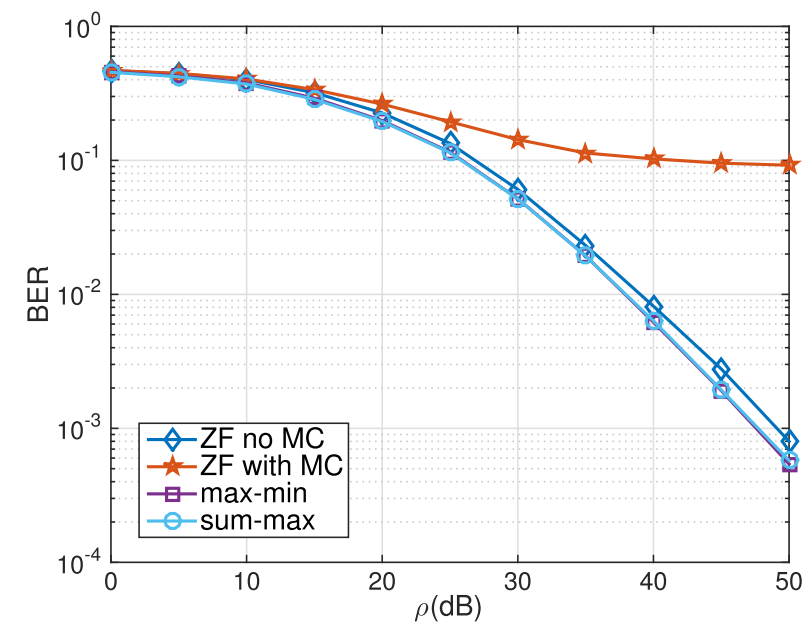

Fig. 6. BER performance of the proposed schemes, $4 \times 4, d_{t}=0.2, d_{r}=0.5$, $d_{l}=0.3,16 \mathrm{QAM}$.

is $F=20$ symbols. For both modulations, it can be observed that "ZF with MC" achieves the lowest throughput performance due to a higher BER. For the proposed schemes, it is shown that they can improve the throughput performance compared to the conventional case with fixed mutual coupling and even outperform "ZF no MC", as the mutual coupling effect is exploited. It is also observed that the scheme based on 'max-min' outperforms 'sum-max' in terms of the average throughput.

In Fig. 6, the BER performance of the proposed schemes for 16QAM is shown with respect to the transmit SNR. A similar trend can be observed and the proposed schemes can still offer performance gains. The schemes based on 'max-min' and 'sum-max' achieve the same performance for 16QAM, and we can observe a 1-2dB gain compared to the ideal ZF case without mutual coupling. We should note that " $\mathrm{ZF}$ no MC" here is only shown as a reference, and the performance gain over the "ZF with MC" in practice is very large at high SNR regime, which means that the proposed schemes are also applicable to QAM modulations. 


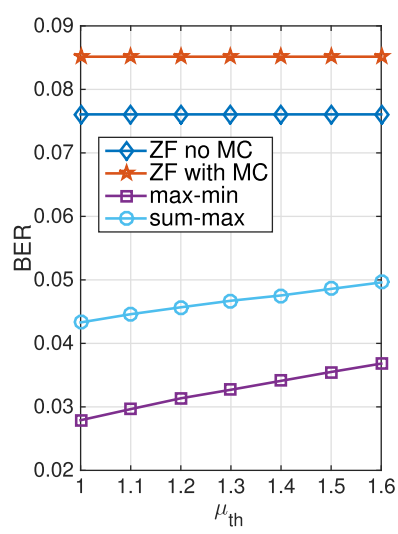

(a) BPSK

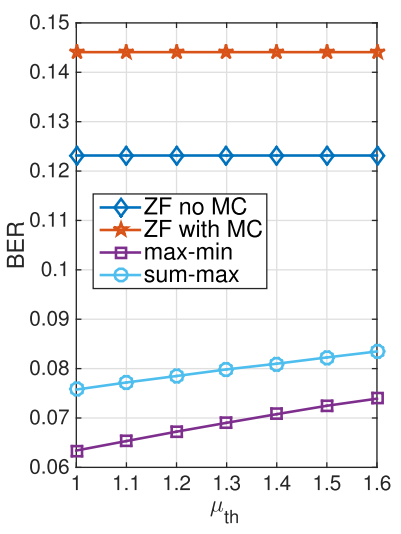

(b) QPSK
Fig. 7. BER performance v.s. $\mu_{t h}, 4 \times 4, d_{t}=0.2, d_{r}=0.5, d_{l}=0.3$, $\mathrm{SNR}=20 \mathrm{~dB}$

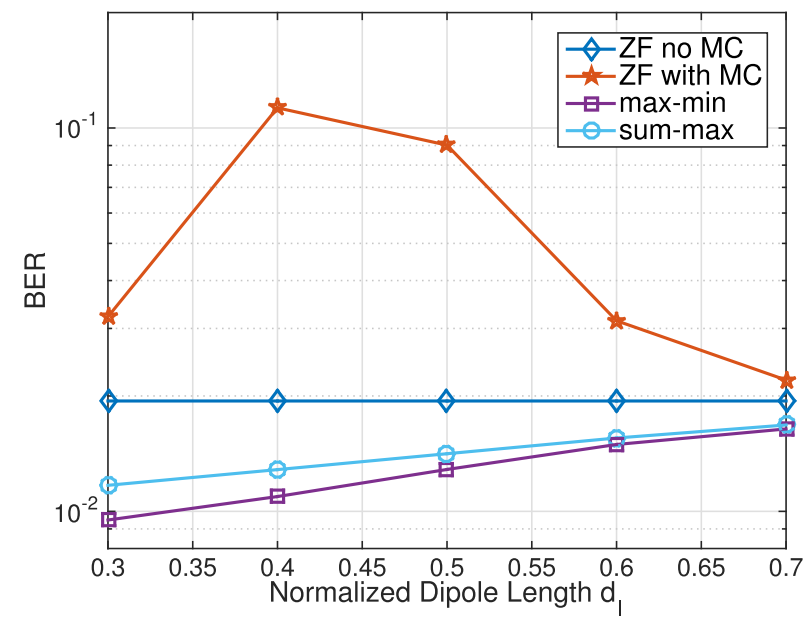

Fig. 8. BER performance v.s. normalized dipole length, $4 \times 4, d_{t}=0.2$, $d_{r}=0.5, \mathrm{SNR}=30 \mathrm{~dB}, \mu_{t h}=1.1$, QPSK

Fig. 7 shows the BER performance of the proposed schemes with respect to the optimization parameter $\mu_{t h}$ for both BPSK and QPSK. For the proposed schemes based on constructive interference, it is seen that with an increase in the value of $\mu_{t h}$, the BER performance becomes slightly worse, which is because a larger value of $\mu_{t h}$ reduces the value range of the optimal solutions by convex optimization, as seen in $\mathcal{P}_{3}$ and $\mathcal{P}_{5}$. Moreover, a consistent performance gain can be observed for 'max-min' over 'sum-max'.

In the following simulations, without loss of generality we focus on the QPSK modulation. In Fig. 8, the BER performance is compared with respect to the normalized dipole length. As can be seen, for "ZF with MC", when the dipole length is equal to 0.4 , the mutual coupling effect is the most severe and greatly degrade the BER performance. The performance of "ZF no MC" does not depend on the dipole length and therefore remain unchanged for all values of the dipole length. For the proposed schemes, it is observed that they outperform both "ZF with MC" and "ZF no MC" with the exploitation of mutual coupling. Generally, techniques based on 'max-min' achieve the best performance for all values of

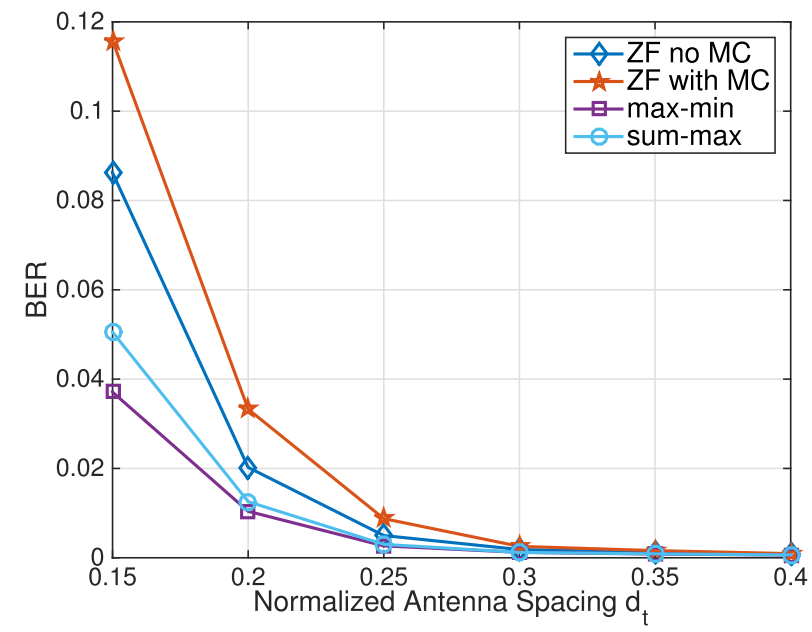

Fig. 9. BER performance v.s. normalized antenna spacing at the transmitter, $4 \times 4, d_{r}=0.5, d_{l}=0.3, \mathrm{SNR}=30 \mathrm{~dB}, \mu_{t h}=1.1$, QPSK

dipole length. It can also be seen that with the increase in the dipole length, the BER performance becomes slightly worse, and this is because when the dipole length increases, $\Re\left(z_{A}\right)$ becomes larger, which then reduces the possible value range of each tunable load and therefore leads to a worse BER performance.

Fig. 9 compares the BER performance of the proposed schemes with an increase in the antenna spacing at the transmitter. As can be observed, when the antenna spacing is small, "ZF with MC" achieves a much worse BER performance compared to other schemes due to the strong mutual coupling and correlation effect among the antenna array. For the proposed schemes, it is observed that they outperform "ZF with MC" and even "ZF no MC" as the mutual coupling effect is exploited to further benefit the system performance, where 'max-min' achieves the best performance. With the increase in the antenna spacing, the performance gain between the proposed schemes and "ZF with MC" becomes smaller as the mutual coupling effect becomes weak, while the BER performance of all schemes is improved, which is due to the reduced correlation effect when the antenna spacing is increased. The proposed schemes therefore enable the design of compact antenna arrays.

Fig. 10 presents the BER performance with respect to the number of transmit antennas where we assume $N_{r}=N_{t}$ at $\mathrm{SNR}=30 \mathrm{~dB}$. With an increase in the antenna number, while the BER performance becomes worse for each scheme, a consistent performance gain can be observed for the proposed schemes over conventional ZF receiver with a fixed mutual coupling. The performance gain of the proposed schemes persists with the number of antennas increasing. Moreover, for all cases it is observed that the proposed scheme based on 'max-min' criterion outperforms that with 'sum-max'.

Fig. 11 presents the feasibility of the 'max-min' optimization $\mathcal{P}_{3}$ with respect to the number of antennas where the upper bound is obtained by (71). As can be seen, with an increase in the optimization parameter $\mu_{t h}$, the feasibility improves while the performance will be degraded, as evidenced by Fig. 7, 


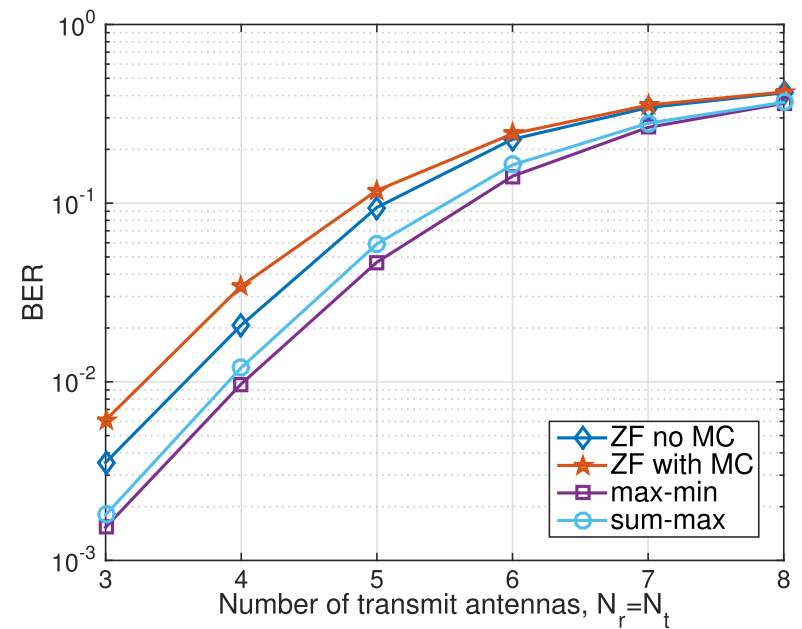

Fig. 10. BER performance v.s. antenna number, $N_{r}=N_{t}, d_{t}=0.2$, $d_{r}=0.5, d_{l}=0.3, \mathrm{SNR}=30 \mathrm{~dB}, \mu_{t h}=1.1$, QPSK

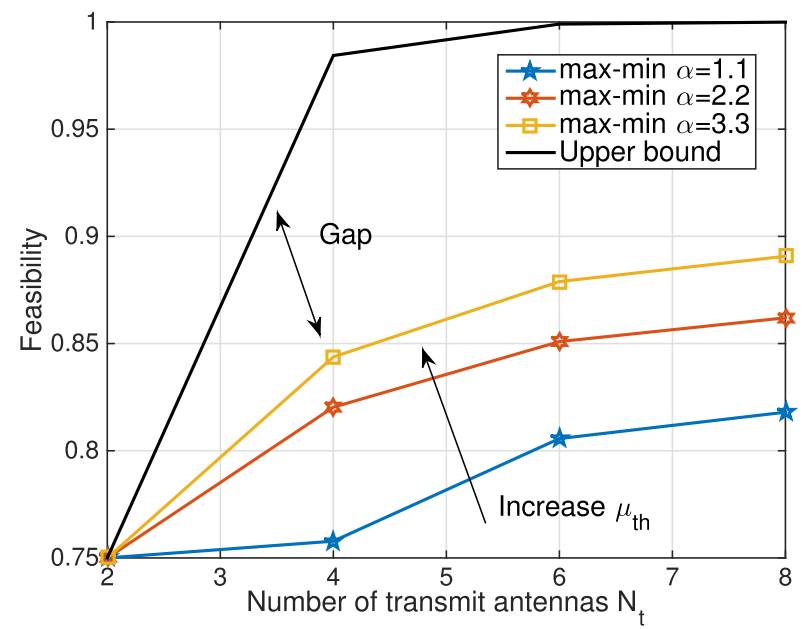

Fig. 11. Feasibility of optimization v.s. antenna number, $N_{r}=N_{t}, d_{t}=0.2$, $d_{r}=0.5, d_{l}=0.3$, QPSK

which means there exists a tradeoff between the performance and the feasibility.

\section{CONCLUSION}

In this paper, the mutual coupling effect among antenna elements is investigated and exploited for P2P MIMO systems. While it is shown that it is not possible to fully eliminate the effect of mutual coupling solely by changing the antenna load impedance values, schemes that exploit the mutual coupling to further benefit the performance are proposed. By formulating the problem into a convex optimization problem to obtain the optimal load impedance values with practical constraints considered, we manipulate the mutual coupling effect to achieve phase alignment where the constructive region is exploited. The implementation of the proposed schemes is also discussed, where a lookup table can be introduced for practical application. The simulation results show that the proposed schemes can achieve performance gains over conventional systems with fixed mutual coupling, especially in compact antenna deployments where the spacing between antenna elements is small.

\section{APPENDIX}

\section{CALCUlation of the Antenna ImPEDANCE $Z_{A}$ AND THE MUTUAL IMPEDANCE $Z_{m_{k}}$}

Based on [25], the antenna impedance $z_{A}$ and the mutual impedance $z_{m_{k}}$ can be calculated with EMF methods based on the normalized antenna spacing at the transmitter $d_{t}$. For the antenna impedance, the real part and imaginary part can be calculated respectively as

$$
Z_{z_{A}}=R_{z_{A}}+j \cdot X_{z_{A}}
$$

where $R_{z_{A}}$ is the resistance and $X_{z_{A}}$ is the reactance. The calculation of $R_{z_{A}}$ and $X_{z_{A}}$ is shown in (74) on the bottom of this page, where $\eta=120 \pi$ is the intrinsic impedance, $C_{i}$ and $S_{i}$ denote the cosine integral function and sine integral function, respectively. $\gamma_{0}$ is the Euler constant, $K=\frac{2 \pi}{\lambda_{0}}$, and $L=d_{l} \cdot \lambda_{0}$, where $\lambda_{0}$ is the carrier wavelength. $a$ is the radius of the wire and a typical value $a=0.001 \mathrm{~m}$ is applied in this paper.

For a uniform linear dipole antenna array with side-by-side configuration, as assumed in this paper, the mutual impedance can be calculated as

$$
z_{m_{k}}=R_{m_{k}}+j \cdot X_{m_{k}} .
$$

In (75), $R_{m_{k}}$ and $X_{m_{k}}$ can be calculated respectively as

$$
\begin{aligned}
R_{m_{k}} & =\frac{\eta}{4 \pi}\left\{2 C_{i}\left[u_{0}(k)\right]-C_{i}\left[u_{1}(k)\right]-C_{i}\left[u_{2}(k)\right]\right\}, \\
X_{m_{k}} & =-\frac{\eta}{4 \pi}\left\{2 S_{i}\left[u_{0}(k)\right]-S_{i}\left[u_{1}(k)\right]-S_{i}\left[u_{2}(k)\right]\right\},
\end{aligned}
$$

$$
\begin{aligned}
& R_{z_{A}}=\frac{\eta}{2 \pi \sin ^{2}\left(\frac{K L}{2}\right)}\left\{\gamma_{0}+\ln (K L)-C_{i}(K L)+\frac{\sin (K L)}{2} \cdot\left[S_{i}(2 K L)-2 S_{i}(K L)\right]\right. \\
& \left.+\frac{\cos (K L)}{2}\left[\gamma_{0}+\ln \left(\frac{K L}{2}\right)+C_{i}(2 K L)-2 C_{i}(K L)\right]\right\}, \\
& X_{z_{A}}=\frac{\eta}{4 \pi \sin ^{2}\left(\frac{K L}{2}\right)}\left\{2 S_{i}(K L)+\cos (K L) \cdot\left[2 S_{i}(K L)-S_{i}(K L)\right]\right. \\
& \left.-\sin (K L) \cdot\left[2 C_{i}(K L)-C_{i}(2 K L)-C_{i}\left(\frac{2 K a^{2}}{L}\right)\right]\right\} .
\end{aligned}
$$


where $u_{0}(k), u_{1}(k)$ and $u_{2}(k)$ can be calculated as

$$
\begin{aligned}
& u_{0}(k)=2 \pi \cdot k d_{t}, \\
& u_{1}(k)=2 \pi \cdot\left(\sqrt{\left(k d_{t}\right)^{2}+d_{l}^{2}}+d_{l}\right), \\
& u_{2}(k)=2 \pi \cdot\left(\sqrt{\left(k d_{t}\right)^{2}+d_{l}^{2}}-d_{l}\right) .
\end{aligned}
$$

\section{REFERENCES}

[1] D. Gesbert, M. Shafi, D. Shiu, P. J. Smith, and A. Naguib, "From theory to practice: An overview of MIMO space-time coded wireless systems," IEEE J. Sel. Areas Commun., vol. 21, no. 3, pp. 281-302, Apr. 2003.

[2] A. J. Paulraj, D. A. Gore, R. U. Nabar, and H. Bolcskei, "An overview of MIMO communications-A key to gigabit wireless," Proc. IEEE, vol. 92, no. 2, pp. 198-218, Feb. 2004.

[3] K.-K. Wong and A. J. Paulraj, "On the decoding order of MIMO maximum-likelihood sphere decoder: Linear and non-linear receivers," in Proc. IEEE 59th Veh. Technol. Conf. (VTC), vol. 2. May 2007, pp. 698-702.

[4] C. Wang, E. K. S. Au, R. D. Murch, W. H. Mow, R. S. Cheng, and V. Lau, "On the performance of the MIMO zero-forcing receiver in the presence of channel estimation error," IEEE Trans. Wireless Commun., vol. 6, no. 3, pp. 805-810, Mar. 2007.

[5] M. R. McKay, I. B. Collings, and A. M. Tulino, "Achievable sum rate of MIMO MMSE receivers: A general analytical framework," IEEE Trans. Inf. Theory, vol. 56, no. 1, pp. 396-410, Jan. 2010.

[6] C. Masouros, "Correlation rotation linear precoding for MIMO broadcast communications," IEEE Trans. Signal Process., vol. 59, no. 1, pp. 252-262, Jan. 2011.

[7] S. M. Razavi, T. Ratnarajah, and C. Masouros, "Transmit-power efficient linear precoding utilizing known interference for the multiantenna downlink," IEEE Trans. Veh. Technol., vol. 63, no. 9, pp. 4383-4394, Nov. 2014.

[8] M. Alodeh, S. Chatzinotas, and B. Ottersten, "Constructive multiuser interference in symbol level precoding for the MISO downlink channel," IEEE Trans. Signal Process., vol. 63, no. 9, pp. 2239-2252, May 2015.

[9] C. Masouros and G. Zheng, "Exploiting known interference as green signal power for downlink beamforming optimization," IEEE Trans. Signal Process., vol. 63, no. 14, pp. 3628-3640, Jul. 2015.

[10] S. Biswas, C. Masouros, and T. Ratnarajah, "Performance analysis of large multiuser MIMO systems with space-constrained 2-D antenna arrays," IEEE Trans. Wireless Commun., vol. 15, no. 5, pp. 3492-3505, May 2016.

[11] C. Masouros, M. Sellathurai, and T. Ratnarajah, "Large-scale MIMO transmitters in fixed physical spaces: The effect of transmit correlation and mutual coupling," IEEE Trans. Commun., vol. 61, no. 7, pp. 2794-2804, Jul. 2013.

[12] M. T. Ivrlac, W. Utschick, and J. A. Nossek, "Fading correlations in wireless MIMO communication systems," IEEE J. Sel. Areas Commun., vol. 21, no. 5, pp. 819-828, Jun. 2003.

[13] D. Piazza, N. J. Kirsch, A. Forenza, R. W. Heath, and K. R. Dandekar, "Design and evaluation of a reconfigurable antenna array for MIMO systems," IEEE Trans. Antennas Propag., vol. 56, no. 3, pp. 869-881, Mar. 2008.

[14] P. L. Kafle, A. Intarapanich, A. B. Sesay, J. Mcrory, and R. J. Davies, "Spatial correlation and capacity measurements for wideband MIMO channels in indoor office environment," IEEE Trans. Wireless Commun., vol. 7, no. 5, pp. $1560-1571$, May 2008 .

[15] A. M. Tulino, A. Lozano, and S. Verdu, "Impact of antenna correlation on the capacity of multiantenna channels," IEEE Trans. Inf. Theory, vol. 51, no. 7, pp. 2491-2509, Jul. 2005.

[16] G. Alfano, A. M. Tulino, A. Lozano, and S. Verdu, "Capacity of MIMO channels with one-sided correlation," in Proc. IEEE Conf. Spread Spectr. Techn. Appl., Aug./Sep. 2004, pp. 515-519.

[17] M. Kiessling and J. Speidel, "Analytical performance of MIMO zeroforcing receivers in correlated Rayleigh fading environments," in Proc. 4th IEEE Workshop Signal Process. Adv. Wireless Commun. (SPAWC), Jun. 2003, pp. 383-387.

[18] H. Liu, Y. Song, and R. C. Qiu, "The impact of fading correlation on the error performance of MIMO systems over Rayleigh fading channels," IEEE Trans. Wireless Commun., vol. 4, no. 5, pp. 2014-2019, Sep. 2005.
[19] C.-K. Wen, Y.-N. Lee, J.-T. Chen, and P. Ting, "Asymptotic spectral efficiency of MIMO multiple-access wireless systems exploring only channel spatial correlations," IEEE Trans. Signal Process., vol. 53, no. 6 , pp. 2059-2073, Jun. 2005.

[20] A. Alexiou and M. Qaddi, "Robust linear precoding to compensate for antenna correlation in orthogonal space-time block coded systems," in Proc. Sensor Array Multichannel Signal Process. Workshop, Jul. 2004, pp. 701-705.

[21] H. R. Bahrami and T. Le-Ngoc, "Precoder design based on the channel correlation matrices," IEEE Trans. Wireless Commun., vol. 5, no. 12, pp. 3579-3587, Dec. 2006.

[22] J. Akhtar and D. Gesbert, "Spatial multiplexing over correlated MIMO channels with a closed-form precoder," IEEE Trans. Wireless Commun., vol. 4, no. 5, pp. 2400-2409, Sep. 2005.

[23] B. Clerckx, C. Craeye, D. Vanhoenacker-Janvier, and C. Oestges, "Impact of antenna coupling on $2 \times 2$ MIMO communications," IEEE Trans. Veh. Technol., vol. 56, no. 3, pp. 1009-1018, May 2007.

[24] S. Lu, H. T. Hui, M. E. Bialkowski, X. Liu, H. S. Hui, and N. V. Shuley, "Effects of antenna mutual coupling on the performance of MIMO systems," in Proc. 29th Symp. Inf. Theory, Benelux, Belgium, May 2008, pp. 2945-2948.

[25] C. A. Balanis, Antenna Theory: Analysis and Design, 4th ed. Hoboken, NJ, USA: Wiley, Mar. 2016.

[26] I. J. Gupta and A. A. Ksienski, "Effect of mutual coupling on the performance of adaptive arrays," IEEE Trans. Antennas Propag., vol. 31, no. 5, pp. 785-791, Sep. 1983

[27] A. A. Abouda and S. G. Haggman, "Effect of mutual coupling on capacity of MIMO wireless channels in high SNR," Prog. Electromagn. Res., vol. 65, pp. 27-40, Jun. 2005.

[28] H. Steyskal and J. S. Herd, "Mutual coupling compensation in small array antennas," IEEE Trans. Antennas Propag., vol. AP-38, no. 12, pp. 1971-1975, Dec. 1990.

[29] J. Corcoles, M. A. Gonzalez, and J. Rubio, "Mutual coupling compensation in arrays using a spherical wave expansion of the radiated field," IEEE Antennas Wireless Propag. Lett., vol. 8, pp. 108-111, 2009.

[30] J. Rubio, J. F. Izquierdo, and J. Córcoles, "Mutual coupling compensation matrices for transmitting and receiving arrays," IEEE Trans. Antennas Propag., vol. 63, no. 2, pp. 839-843, Feb. 2015.

[31] G. Moreno, H. M. Bernety, and A. B. Yakovlev, "Reduction of mutual coupling between strip dipole antennas at terahertz frequencies with an elliptically shaped graphene monolayer," IEEE Ant. Wireless Propag. Lett., vol. 15, pp. 1533-1536, Dec. 2015.

[32] S. Farsi, H. Aliakbarian, D. Schreurs, B. Nauwelaers, and G. A. E. Vandenbosch, "Mutual coupling reduction between planar antennas by using a simple microstrip U-section," IEEE Antennas Wireless Propag. Lett., vol. 11, pp. 1501-1503, 2012.

[33] Z. Li, Z. Du, M. Takahashi, K. Saito, and K. Ito, "Reducing mutual coupling of MIMO antennas with parasitic elements for mobile terminals," IEEE Trans. Ant. Propag., vol. 60, no. 2, pp. 473-481, Feb. 2012.

[34] H. T. Hui, "A practical approach to compensate for the mutual coupling effect in an adaptive dipole array," IEEE Trans. Antennas Propag., vol. 52, no. 5, pp. 1262-1269, May 2004.

[35] I. Salonen, A. Toropainen, and P. Vainikainen, "Linear pattern correction in a small microstrip antenna array," IEEE Trans. Antennas Propag., vol. 52, no. 2, pp. 578-586, Feb. 2004.

[36] R. S. Adve and T. K. Sarkar, "Compensation for the effects of mutual coupling on direct data domain adaptive algorithm," IEEE Trans. Antennas Propag., vol. 48, no. 1, pp. 86-94, Jan. 2000.

[37] D. Feng, L. Lu, Y. Yuan-Wu, G. Li, S. Li, and G. Feng, "Deviceto-device communications in cellular networks," IEEE Commun. Mag., vol. 52, no. 4, pp. 49-55, Apr. 2014

[38] J. Hoydis, M. Kobayashi, and M. Debbah, "Green small-cell networks," IEEE Veh. Technol. Mag., vol. 6, no. 1, pp. 37-43, Mar. 2011.

[39] J. G. Andrews et al., "What will 5G be?" IEEE J. Sel. Areas Commun., vol. 32, no. 6, pp. 1065-1082, Jun. 2014.

[40] A. M. Sayeed, "Deconstructing multiantenna fading channels," IEEE Trans. Signal Process., vol. 50, no. 10, pp. 2563-2579, Oct. 2002.

[41] M. Steinbauer, A. F. Molisch, and E. Bonek, "The double-directional radio channel," IEEE Antennas Propag. Mag., vol. 43, no. 4, pp. 51-63, Aug. 2001.

[42] A. Forenza, D. J. Love, and R. W. Heath, Jr., "Simplified spatial correlation models for clustered MIMO channels with different array configurations," IEEE Trans. Veh. Technol., vol. 56, no. 4, pp. 1924-1934, Jul. 2007. 
[43] J. Choma and W. K. Chen, Feedback Networks: Theory and Circuit Applications. Hackensack, NJ, USA: World Scientific Publishing, May 2007.

[44] H. A. Haus, Electromagnetic Noise and Quantum Optical Measurements. Springer, Nov. 2000.

[45] C. Masouros, T. Ratnarajah, M. Sellathurai, C. B. Papadias, and A. K. Shukla, "Known interference in the cellular downlink: A performance limiting factor or a source of green signal power?" IEEE Commun. Mag. vol. 51, no. 10, pp. 162-171, Oct. 2013.

[46] M. Alodeh, S. Chatzinotas, and B. Ottersten, "Energy-efficient symbollevel precoding in multiuser MISO based on relaxed detection region," IEEE Trans. Wireless Commun., vol. 15, no. 5, pp. 3755-3767, Feb. 2016.

[47] M. Alodeh, S. Chatzinotas, and B. Ottersten, "Constructive interference through symbol level precoding for multi-level modulation," in Proc. IEEE Global Commun. Conf. (GLOBECOM), San Diego, CA, USA, Dec. 2015 , pp. 1-6.

[48] M. Alodeh, S. Chatzinotas, and B. Ottersten. "Symbol-level multiuser MISO precoding for multi-level adaptive modulation: A multicast view." [Online]. Available: https://arxiv.org/abs/1601.02788

[49] D. Kwon, W. Y. Yeo, and D. K. Kim, "A new precoding scheme for constructive superposition of interfering signals in multiuser MIMO systems," IEEE Commun. Lett., vol. 18, no. 11, pp. 2047-2050, Nov. 2014.

[50] D. Kwon, H. S. Kang, and D. K. Kim, "Robust interference exploitationbased precoding scheme with quantized CSIT," IEEE Commun. Lett., vol. 20, no. 4, pp. 780-783, Apr. 2016.

[51] R. Xu and F. C. M. Lau, "Performance analysis for MIMO systems using zero forcing detector over fading channels," IEE Proc. Commun., vol. 153, no. 1, pp. 74-80, Feb. 2006.

[52] I. S. Gradshteyn and I. M. Ryzhik, Table of Integral, Series and Products, 6th ed. San Diego, CA, USA: Academic, 2000.

[53] J.-S. Fu, "Adaptive Impedance Matching Circuits based on Ferroelectric and Semiconductor Varactors," Ph.D. dissertation, Dept. Elect. Eng., Univ. Michigan, Ann Arbor, MI, USA, 2009.

[54] R. York et al., "Microwave integrated circuits using thin-film BST," in Proc. 12th Аnnu. Int. Symp. Appl. Ferroelectr., vol. 1. Jul./Aug. 2000, pp. 195-200.

[55] J. Lu, D. Ireland, and R. Schlub, "Dielectric embedded ESPAR (DE-ESPAR) antenna array for wireless communications," IEEE Trans. Antennas Propag., vol. 53, no. 8, pp. 2437-2443, Aug. 2005.

[56] B. Alshami, H. Aboulnour, and M. Dib, "Design of a broadband ESPAR antenna," in Proc. Microw. Symp. Mediterrannean (MMS), Nov. 2009, pp. 1-6.

[57] O. N. Alrabadi, C. B. Papadias, A. Kalis, N. Marchetti, and R. Prasad, "MIMO transmission and reception techniques using three-element ESPAR antennas," IEEE Commun. Lett., vol. 13, no. 4, pp. 236-238, Apr. 2009.

[58] L. Zhou, F. A. Khan, T. Ratnarajah, and C. B. Papadias, "Achieving arbitrary signals transmission using a single radio frequency chain," IEEE Trans. Commun., vol. 63, no. 12, pp. 4865-4878, Oct. 2015.

[59] G. C. Alexandropoulos, V. I. Barousis, and C. B. Papadias, "Precoding for multiuser MIMO systems with single-fed parasitic antenna arrays," in Proc. IEEE Global Commun. Conf. (GLOBECOM), Dec. 2014, pp. 3897-3902.
[60] V. I. Barousis and C. B. Papadias, "Arbitrary precoding with single-fed parasitic arrays: Closed-form expressions and design guidelines," IEEE Wireless Commun. Lett., vol. 3, no. 2, pp. 229-232, Feb. 2014.

[61] O. N. Alrabadi et al., "Spatial multiplexing with a single radio: Proofof-concept experiments in an indoor environment with a $2.6-\mathrm{GHz}$ prototype," IEEE Commun. Lett., vol. 15, no. 2, pp. 178-180, Feb. 2011.

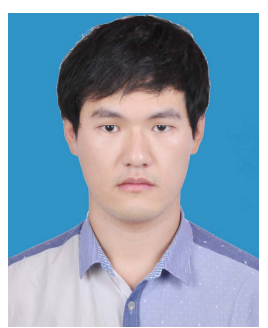

Ang Li (S'14) received the bachelor's and master's degrees in electronic and information engineering from Xi'an Jiaotong University, in 2011 and 2014, respectively. $\mathrm{He}$ is currently pursuing the $\mathrm{Ph} . \mathrm{D}$. degree with the Communications and Information Systems Research Group, Department of Electronic and Electrical Engineering, University College London. His research interests lie in the field of wireless communications with focus on beamforming designs for MIMO systems and mmWave communications.

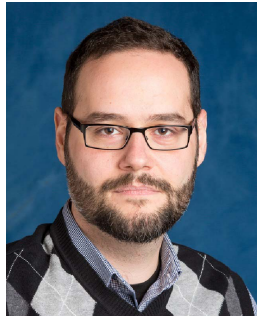

Christos Masouros (M'06-SM'14) received the Diploma degree in electrical and computer engineering from the University of Patras, Greece, in 2004, and the M.Sc. degree in Research and the Ph.D. degree in electrical and electronic engineering from the University of Manchester, Manchester, U.K., in 2006 and 2009, respectively. In 2008, he was a Research Intern with the Philips Research Laboratories, U.K. From 2009 to 2010, he was a Research Associate with the University of Manchester, and from 2010 to 2012 he was a Research Fellow with Queen's University Belfast. He held the Royal Academy of Engineering Research Fellowship from 2011 to 2016.

$\mathrm{He}$ is currently a Senior Lecturer with the Communications and Information Systems Research Group, Department of Electronic and Electrical Engineering, University College London. His research interests lie in the field of wireless communications and signal processing with particular focus on green communications, large scale antenna systems, cognitive radio, interference mitigation techniques for MIMO, and multicarrier communications. He was a recipient of the Best Paper Award at the IEEE GlobeCom Conference held in 2015, and has been recognized as an Exemplary Editor of the IEEE COMMUNICATIONS LeTtERs, and as an Exemplary Reviewer of the IEEE TRANSACTIONS ON COMMUNiCATIONS. He is an Associate Editor of the IEEE COMMUNICATIONS LETTERS, and Guest Editor of the IEEE JOURNAL on Selected Topics in Signal Processing Special Issue on Exploiting Interference Towards Energy Efficient and Secure Wireless Communications. 